\title{
AN INVENTORY PROBLEM WITH TWO RANDOMLY AVAILABLE SUPPLIERS
}

\author{
ÜLKÜ GÜRLER
}

Bilkent University, Bilkent/Ankara, Turkey

\author{
MAHMUT PARLAR
}

McMaster University, Hamilton, Ontario, Canada

(Received June 1993; revisions received May 1994, January 1995, July 1995; accepted February 1996)

\begin{abstract}
This paper considers a stochastic inventory model in which supply availability is subject to random fluctuations that may arise due to machine breakdowns, strikes, embargoes, etc. It is assumed that the inventory manager deals with two suppliers who may be either individually ON (available) or OFF (unavailable). Each supplier's availability is modeled as a semi-Markov (alternating renewal) process. We assume that the durations of the ON periods for the two suppliers are distributed as Erlang random variables. The OFF periods for each supplier have a general distribution. In analogy with queuing notation, we call this an $E_{s_{1}}\left[E_{s_{2}}\right] / G_{1}\left[G_{2}\right]$ system. Since the resulting stochastic process is non-Markovian, we employ the "method of stages" to transform the process into a Markovian one, albeit at the cost of enlarging the state space. We identify the regenerative cycles of the inventory level process and use the renewal reward theorem to form the long-run average cost objective function. Finite time transition functions for the semi-Markov process are computed numerically using a direct method of solving a system of integral equations representing these functions. A detailed numerical example is presented for the $E_{2}\left[E_{2}\right] / M[M]$ case. Analytic solutions are obtained for the particular case of "large" (asymptotic) order quantity, in which case the objective function assumes a very simple form that can be used to analyze the optimality conditions. The paper concludes with the discussion of an alternative inventory policy for modeling the random supply availability problem.
\end{abstract}

$\mathrm{O}$ ne of the unstated assumptions in almost every inventory model is the continuous availability of the supply at any time an order is placed. Even if there is a (possibly random) lead time, it is assumed that the supplier will immediately start working on the order and will deliver the required quantity when the lead time ends.

However, supply of products may be disrupted due to several reasons, as we will discuss below. Thus, in a general setup the supplier could be considered to have ON and OFF times with random durations. Such disruptions could be induced by various factors, depending on the underlying structure of the model, and the interpretation of the ON/ OFF times would follow accordingly.

As a general example, we may consider the supplier with its own inventory process, and we could say that the supplier is ON if our order quantity $q$ is available in its inventory, and is OFF otherwise. As another example that could be encountered frequently, consider a supplier as a production process that is under statistical process control. From time to time, the process starts to produce items out of specification limits beyond an acceptable proportion, and the process is halted to reach the desired capability. The OFF times of the supplier would then correspond to the termination of production for system inspection. Machine breakdowns or preventive maintenance policies could also result in disruptions in the production process, which in turn could induce supplier unavailability for the inventory process under study.
As in the above examples, when the supply may be cut off at random times for random durations, the implicit assumption of continuous supply availability would no longer be valid, and new models would be necessary to incorporate the disruptions of supply. Especially with the recent emphasis on the just-in-time replenishment systems with a number of suppliers, such models may be useful for production managers.

Silver (1981) appears to be the first author to discuss the need for models that deal with supplier uncertainty. (For additional comments on this type of uncertainty, see the text by Nahmias (1993, p. 186).) In a recent paper Parlar and Berkin (1991) consider the supplier uncertainty problem for the classical economic order quantity (EOQ) model, assuming that there is a single supplier whose ON and OFF periods are distributed exponentially. They fix the reorder point at zero and, using the renewal reward theorem (RRT), find the optimal order quantity. Parlar and Perry (1996) extend the EOQ model to include multiple unreliable suppliers whose $\mathrm{ON} / \mathrm{OFF}$ periods are all distributed exponentially with different parameters. They assume that unplanned shortages due to supplier unavailability are backordered. Probabilistic analysis of the model is conducted using the properties of the continuous time Markov chain (CTMC) representing the suppliers' status. Gupta (1996) analyzes a continuous review, order quantity/ reorder point inventory system with an unreliable supplier whose ON/OFF periods are distributed exponentially. $\mathrm{He}$ 
assumes that the unit demands are generated according to a Poisson process, and the shortages result in lost sales. Moinzadeh and Aggarwal (1992) study a production/inventory system, i.e., the classical production run size model, subject to random disruptions, assuming constant demand and constant production rate. In another recent paper, Parlar (1997) considers a continuous-review problem with an unreliable supplier whose delivery lead time (when available) is also random. He generalizes the assumption regarding the unreliable supplier's ON/OFF periods, and assumes that the ON period is distributed as $E_{s}$ ( $s$-stage Erlangian), and the OFF period has a general distribution. (In analogy with queuing notation, this is called an $E_{s} / G$ system.)

In this paper, we generalize some of the above results in several directions. First, we assume that the inventory manager deals with two suppliers. Both of these suppliers may be $\mathrm{ON}$ or $\mathrm{OFF}$ at different times for random durations. As to the application of the above ideas to a system with two suppliers, consider the following examples: (1) two parallel machines supplying raw material to a third one, which processes the raw material at a constant rate. The inventory problem here relates to the amount of the raw material that would be kept in the buffer of the third machine. As mentioned above, the suppliers' OFF times could correspond to the inadequate amount of raw materials in the buffers of the first two machines due to machine breakdowns, power cuts, etc.; and (2) A similar approach can be adopted for a production plant operating under JIT with two vendors. Note here that in JIT systems the number of vendors are kept minimal, as opposed to the traditional systems in which the number of vendors are maximized to reduce the price by competition. Therefore, the two-supplier assumption appears applicable. The OFF times of the vendors again may arise due to reasons explained above. For other reasons (such as high inflation and embargoes) that may result in supply disruptions, see Silver (1981), Chao (1987), and Chao et al. (1989). A twosupplier market with unreliable suppliers could be a good model for imported goods that may be supplied mainly by two countries. For example, the United States and Japan are the two main suppliers for many products such as computers, communications systems, etc., which require high technological expertise. For various reasons, supply of these products may be disrupted. A temporary shift of production to other items, increased exports to other countries for political reasons, reduction of production due to local economic factors, could be cited among them.

Alternating renewal process models are conveniently used as a framework for the representation of such disruptive events for which the arrival time and duration are uncertain. In specific applications for which the distributions of these periods are required, exponential distribution is commonly used (Meyer et al. 1979, Parlar and Berkin 1991), which simplifies the solution. As a second generalization of the previously mentioned results, in the present study we allow quite general forms for the $\mathrm{ON} /$
OFF periods. In particular, the distributions of the ON periods for suppliers 1 and 2 are assumed Erlangian with $s_{1}$ and $s_{2}$ stages, respectively, i.e., they are $E_{s_{1}}$ and $E_{s_{2}}$. The distribution of the OFF periods is assumed to be general, i.e., $G_{1}$ and $G_{2}$ for the two suppliers. Analogous to the notation used in queuing theory, we denote this system by $E_{s_{1}}\left[E_{s_{2}}\right] / G_{1}\left[G_{2}\right]$. These assumptions make the ON/OFF stochastic process rather general, since any nonexponential random variable with coefficient of variation (COV) less than unity can be approximated by an Erlang random variable with a proper choice of the stage parameter of the Erlang (Kleinrock 1975).

Finally, for the two-supplier case studied in the present paper, the superposition of two different alternating processes further complicates the technical structure of the model, as opposed to the single-supplier models studied in Parlar and Berkin (1991) and Parlar (1997). This also leads to complications in the solution of the model, as will be seen in the numerical computations to be presented in Section 2.1.

In Section 1, we introduce the model and discuss the application of renewal reward theorem (RRT) in the formulation of the objective function. Computation of the expected cycle length and cycle cost requires the exact solution of the transition functions of the semi-Markov process (SMP) representing the ON/OFF process induced by the two suppliers' availability distributions. We provide the transient (finite time) solution of the transition functions of the SMP using a direct method of solving integral equations representing these functions. In Section 2, we consider a particular case of the model in which $s_{1}=s_{2}=$ 2 . We discuss another case, assuming that the optimal order quantity $q$ is asymptotically "large" compared to the demand rate. This assumption reduces the computational requirements, since only the limiting probabilities of the SMP are required in the analysis of the cost function. It is shown that the objective function assumes a very simple form that can be easily analyzed. The paper concludes with a summary and possible extensions of the current research.

\section{THE $E_{s_{1}}\left[E_{s_{2}}\right] / G_{1}\left[G_{2}\right]$ MODEL: ERLANGIAN "ON" AND GENERAL "OFF" PERIODS}

\section{a) Assumptions}

Consider the case in which the inventory manager may place his order with either of the two suppliers in the market. Each supplier alternates between ON/OFF intervals independently of each other. The inventory policy used is to order $q$ units from either of the two suppliers when the inventory level drops to a reorder point of $r$ units as long as at least one supplier is available. If neither of the suppliers is available when the reorder point is reached, the manager must wait until one of them becomes available again. Although one can incorporate, for example, the price differences between the suppliers into the model and analyze the diversification issues as in Gerchak and Parlar (1990), we will assume that both suppliers 


\section{Inventory Level}

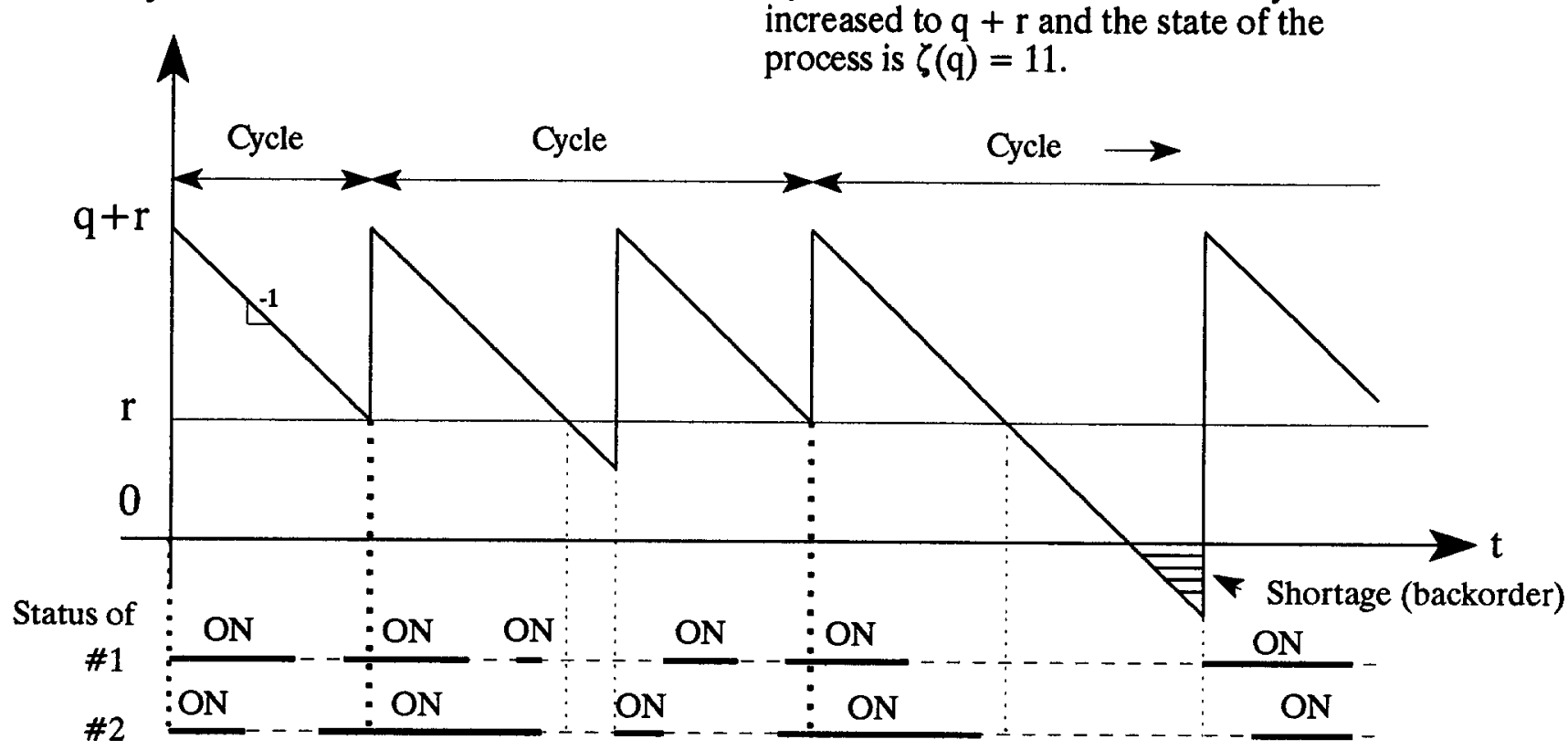

Figure 1. A sample realization of the inventory level process.

are identical, except for a possible difference in the distributions of their ON and OFF durations. In what follows, we assume that demand is deterministic and that all other assumptions of the EOQ model apply (Silver and Petersen, Section 5.1). Without loss of generality, we set the demand rate $D \equiv 1$ "unit"/time. There is an order cost of $\$ K /$ order, holding cost is $\$ h /$ unit/time, and the unit backorder cost is $\$ b /$ unit. Figure 1 depicts a realization of the inventory level process with the above assumptions. The decision problem requires finding the optimal value of the order quantity $q$ and the reorder point $r$ so that the expected long-run average cost of the system is minimized.

We now discuss our initial assumptions regarding the ON/OFF periods of the two suppliers. The duration $X_{1}$ $\left(X_{2}\right)$ of the ON period of supplier $1(2)$ is assumed to be a random variable with distribution function $F_{1}(x)\left[F_{2}(x)\right]$ and with density $f_{1}(x)\left[f_{2}(x)\right]$. Similarly, the duration $Y_{1}$ $\left(Y_{2}\right)$ of the OFF period of supplier 1 (2) has distribution function $G_{1}(y)\left[G_{2}(y)\right]$ with density $g_{1}(y)\left[g_{2}(y)\right]$. When the inventory drops to $r$ and both suppliers are OFF, then the manager must wait (and possibly incur backorder costs) until either one of the suppliers becomes available. We let $\tau_{1}$ and $\tau_{2}$ be the random time until the availability of the first and the second supplier, respectively, from the time the inventory drops to $r$ and both suppliers are found in the OFF state. Since we are analyzing an infinite horizon problem, these random variables are the limiting residual lifetimes (forward recurrence times) of the OFF states, and they have distributions $A_{i}(w)=P\left[\tau_{i} \leqslant w\right]=\int_{0}^{w}$ [1- $\left.G_{i}(y)\right] d y / E\left(Y_{i}\right), i=1,2$ (Ross 1983, p. 68). When the inventory drops to $r$ and both suppliers are OFF, the manager waits a random amount of time given by:

$$
\begin{aligned}
\tau & =\min \left(\tau_{1}, \tau_{2}\right) \\
& = \begin{cases}\tau_{1}, & \text { with probability } \rho=P\left[\tau_{1} \leqslant \tau_{2}\right], \\
\tau_{2}, & \text { with probability } 1-\rho=P\left[\tau_{1}>\tau_{2}\right],\end{cases}
\end{aligned}
$$

until one of the suppliers becomes available. It can easily be shown that the distribution $A(w)=P[\tau \leqslant w]$ of $\tau$ is given in terms of the distributions of $\tau_{1}$ and $\tau_{2}$ as:

$A(w)=1-\left[1-A_{1}(w)\right]\left[1-A_{2}(w)\right]$,

since $P[\tau \leqslant w]=P\left[\min \left(\tau_{1}, \tau_{2}\right) \leqslant w\right]=1-P\left[\tau_{1}>w\right] P\left[\tau_{2}\right.$ $>w]$. Here, we make the assumption that once the supply disruption is over, all the required quantity to bring the inventory level back up to $q+r$ is ordered and delivered immediately. This is a somewhat limiting assumption, since it may take some time for the supplier to return to full production speed after a period of inactivity; hence, deliveries may initially be gradual. However, relaxing this assumption would bring us into the realm of the production run size model with gradual deliveries where delivery rate would be a nonlinear function of time measured from the start of the ON period. Although we do not do it here, it may be possible to model this scenario by an approximation where initial delivery delays can be incorporated into the OFF periods by a suitable adjustment of their distributions.

We can optimize the stochastic inventory system under consideration by making use of the renewal reward theorem (Ross 1983). Using this theorem we identify the regenerative cycles of the inventory process (where the cycle times and cycle costs must all be i.i.d.) and divide the expected cycle cost by the expected cycle length, which gives the average cost objective function. 


\section{GürLer AND PARLAR /}

\section{b) Simple Case of Exponential ON Periods}

Consider first the simple case of exponential ON periods for both suppliers with parameters $\lambda_{t}$, i.e., $F_{t}(x)=1-$ $e^{-\lambda_{1} x}, x \geqslant 0, i=1,2$ and general OFF periods. For this $M_{1}\left[M_{2}\right] / G_{1}\left[G_{2}\right]$ model, let $\zeta_{t}(t)$ denote SMP representing the status of supplier $i=1,2$. Hence, the vector stochastic process $\zeta(t)=\left[\zeta_{1}(t), \zeta_{2}(t)\right]$ representing the availability status of both suppliers will have four states, i.e., the state space $\hat{\mathscr{S}}$ of $\{\zeta(t), t \geqslant 0\}$ will be $\hat{\mathscr{S}}=\{(i, j) \mid(i, j) \in\{1,0\} \times$ $\{1,0\}\}=\{(1,1),(1,0),(0,1),(0,0)\}$, where we use 1 to denote the ON state and 0 to denote the OFF state for a particular supplier. (For notational convenience, we will shorten $(i, j)$ to $i j$ in the subsequent discussion.)

Let $P_{l, k m}(t)=P\{\zeta(t)=k m \mid \zeta(0)=i j\}, i j, k m \in \hat{\mathscr{S}}$ be the transition function of the stochastic process representing the joint status of the suppliers. Referring to Figure 1, we observe that a cycle starts whenever the inventory is raised to $q+r$ and both suppliers are ON, i.e., when $\zeta(t)=11$. Now, if $T_{11}(q)$ is the random cycle length, conditioning on the state observed when inventory drops to $r$, we have:

$T_{11}(q)= \begin{cases}q, & \text { if } \zeta(q)=11 \\ q+T_{l j}(q), & \text { if } \zeta(q)=i j \neq 11 \\ & {\left[\text { with probability } P_{11, l j}(q)\right],}\end{cases}$

where $T_{i j}(q), i j \neq 11$ is the time necessary to complete the cycle if the state is $i j$ when inventory drops to $r$.

We also have, for $i j=00$ :

$T_{00}= \begin{cases}\tau+T_{10}, & {[\text { with probability } \rho],} \\ \tau+T_{01}, & {[\text { with probability } 1-\rho],}\end{cases}$

and, for $i j=10,01$,

$$
T_{l j}=\left\{\begin{array}{lc}
q, & \text { if } \zeta(q)=11 \\
q+T_{k m}, & \text { if } \zeta(q)=k m \in\{10,01\} \\
& {\left[\text { with probability } P_{\imath, k m}(q)\right] .}
\end{array}\right.
$$

Taking expectations and using the results for the finite time solution of transition functions $P_{u, k m}(q)$ which will be discussed in Section 2, one can, in principle, compute the expected cycle length $\bar{T}_{11}(q)=E\left[T_{11}(q)\right]$ for this relatively simple problem. (Expected cycle cost can also be computed in a similar manner.)

When the ON periods are distributed exponentially and a cycle is defined to start whenever the inventory is increased to $q+r$ and both suppliers are $\mathrm{ON}$, one can use the above procedure to compute $\bar{T}_{11}(q)$. However, when the ON periods are not exponential, this modeling convenience disappears, and remaining length of the ON period when inventory drops to $r$ and at least one supplier is available (i.e., when the state is 01,10 or 11 ) is no longer exponential. This means that the length of the new "cycle" depends on how long the suppliers have been available, hence the above formulas can no longer be used. This makes the computation of the expected cycle length (and cost) very difficult. (The reader may recall the difficulties encountered in modeling non-Markovian queues.)

\section{c) Erlangian ON Periods}

One way of dealing with nonexponential random variables is to approximate them with $s$-stage Erlang $\left(E_{s}\right)$ random variables. If the $\mathrm{COV} \leqslant 1$ for a nonexponential $\mathrm{RV} X$, then one can use Erlang's "method of stages" (see, e.g., Kleinrock 1975) and approximate $X$ by an Erlang RV $X^{\prime}$ with parameters $(s, s \lambda)$. Naturally, $X^{\prime}$ is the sum of $s$ exponential RVs, each with mean $1 /(s \lambda)$. It can be shown that $\operatorname{Var}\left(X^{\prime}\right)=1 /\left(s \lambda^{2}\right)$ and $\operatorname{COV}\left(X^{\prime}\right)=1 / \sqrt{s}$; hence by a judicious choice of the parameter $s$, one can approximate $X$ by $X^{\prime}$ with $s$ stages where each stage is exponential. This method has the advantage of transforming a nonexponential RV into one that is the sum of a finite number of exponential RVs; therefore, the standard Markovian analysis can be used to study the stochastic process induced by $X$ (or equivalently, by $X^{\prime}$ ). The trade-off, of course, is the increase in the size of the state space. For example, if $X_{1}$ and $X_{2}$ are Erlang with parameters $\left(2,2 \lambda_{1}\right)$ and $\left(2,2 \lambda_{2}\right)$, respectively, then we would need to consider a total of $(2+1)(2+1)=9$ states in the state space of the ON/ OFF process for both suppliers. (Such a case will be discussed in detail in Section 2.1.)

Let us then assume that $X_{t} \sim \operatorname{Erlang}\left(s_{l}, s_{l} \lambda_{t}\right)$ with $E\left(X_{\imath}\right)$ $=1 / \lambda_{l}, i=1,2$. Using the "method of stages," we model the availability status of the suppliers as a semi-Markov (alternating renewal) process. In particular, we let $\zeta_{1}(t)$ and $\zeta_{2}(t)$ denote the SMPs representing the status of the two suppliers with $\left\{\zeta_{1}(t)=i, i \in \mathscr{Y}_{1}\right\}$ corresponding to the event that supplier 1 is in the $i$ th stage of his ON time Erlang $\left(s_{1}, s_{1} \lambda_{1}\right)$ duration where $\mathscr{S}_{1}=\left\{1,2, \ldots, s_{1}\right\}$. Letting state 0 correspond to the OFF status, we define $\hat{\mathscr{S}}_{1}=$ $\mathscr{S}_{1} \cup\{0\}$. Similar definitions apply to $\left\{\zeta_{2}(t)=j, j \in \mathscr{Y}_{2}\right\}$, $\mathscr{S}_{2}=\left\{1,2, \ldots, s_{2}\right\}$ and $\hat{\mathscr{S}}_{2}=\mathscr{S}_{2} \cup\{0\}$ for the second supplier having an Erlang $\left(s_{2}, s_{2} \lambda_{2}\right)$ ON time distribution. Hence, the overall supplier availability can be expressed as a SMP $\left\{\zeta(t)=\left[\zeta_{1}(t), \zeta_{2}(t)\right], t \geqslant 0\right\}$ with state space $\hat{\mathscr{S}}=$ $\left\{(i, j) \mid i \in \mathscr{Y}_{1}, j \in \hat{\mathscr{Y}}_{2}\right\}$. In other words, $\{\zeta(t)=i j\}$ represents the event where supplier 1 is at the $i$ th stage and supplier 2 is at the $j$ th stage of their ON/OFF durations where $i \in \hat{\mathscr{S}}_{1}$ and $j \in \hat{\mathscr{S}}_{2}$. See Figure 2 for a transition diagram representing the state space for the process $\{\zeta(t)$, $t \geqslant 0\}$ and the direction of possible movement between states.

In the above description, we note that the state space $\hat{\varphi}$ of $\{\zeta(t), t \geqslant 0\}$ has a total of $N=\left(s_{1}+1\right)\left(s_{2}+1\right)$ states corresponding to all possible ON/OFF combinations for the two suppliers. In Section 1.1, when we compute the expected cycle length and the cost, we will need to make use of the finite time transition function $P_{i j, k m}(q)=P\{\zeta(q)$ $=k m \mid \zeta(0)=i j\}$ for $i j, k m \in \hat{9}$.

\subsection{Objective Function}

This subsection develops the objective function of the model using the renewal reward theorem. Expected cycle 


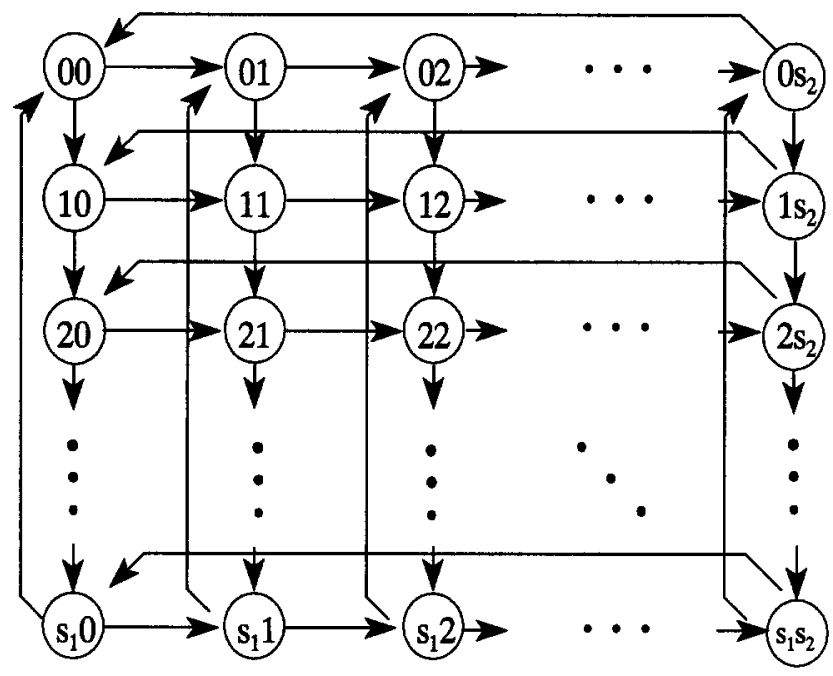

Figure 2. Transitions between states.

length and cycle cost are computed and are then used to form the expected long-run, average cost-objective function. In the computation of the cycle length and cost, it will be convenient to list the $N$-states of the SMP in the following order:

$$
\begin{aligned}
\ell_{N}=[ & 11,12, \ldots, 1 s_{2}, 10\left|21,22, \ldots, 2 s_{2}, 20\right| \\
& \ldots\left|s_{1} 1, s_{1} 2, \ldots, s_{1} s_{2}, s_{1} 0\right| 01,02, \\
& \left.\ldots, 0 s_{2}, 00\right] .
\end{aligned}
$$

\section{a) Cycle Length}

Referring to Figure 1 again, we see that a regenerative cycle for the $E_{s_{1}}\left[E_{s_{2}}\right] / G_{1}\left[G_{2}\right]$ system starts whenever the inventory level is increased to $q+r$ and both suppliers are in the first stage of their Erlangian $\mathrm{ON}$ times, i.e., when $\zeta(t)=11$. (We should note that there may be other alternative definitions of a cycle. For example, we could also define a cycle to start when inventory is raised to $q+r$ units at some time point when the first supplier just commences his ON period and the second supplier is still unavailable.)

Define $T_{11}(q)$ as the random cycle length and let $T_{i j}(q)$, $i j \neq 11$ be the time required to complete the cycle if the process is found in a state $i j$ when the inventory drops to $r$. Here we use $i j \neq 11$ as a shorthand notation for $i j \in$ $\hat{\mathscr{Y}}\left\{\{11\}\right.$. Now, let $\bar{T}_{y j}=E\left[T_{i j}\right], i j \in \hat{\mathcal{S}}$ and define:

$$
\begin{aligned}
\overline{\mathbf{T}}_{(N-1) \times 1}=[ & \bar{T}_{12}, \ldots, \bar{T}_{10}\left|\bar{T}_{21}, \bar{T}_{22}, \ldots, \bar{T}_{20}\right| \\
& \left.\ldots \mid \bar{T}_{01}, \bar{T}_{02}, \ldots, \bar{T}_{00}\right]^{\top},
\end{aligned}
$$

as an $(N-1) \times 1$ column vector of expected subcycle lengths where ' $T$ ' denotes the transpose operator. Define a $1 \times(N-1)$ row vector:

$\mathbf{e}_{1 \times(N-1)}=[, 0, \ldots, \rho|0,0, \ldots, 0|$

$$
\cdots \mid 1-\rho, 0, \ldots, 0] \text {, }
$$

where the only nonzero entries are $\rho$ and $1-\rho$, corresponding to $\bar{T}_{10}$ and $\bar{T}_{01}$, respectively. Also, let $\mathbf{P}$ be the
$N \times N$ matrix of finite time transition functions of the SMP of the suppliers' availability with the row and column elements ordered as in $\ell_{N}$. (In the subsequent discussion, for notational simplicity we will drop the dimension subscripts of $\ell$, e, and $\overline{\mathbf{T}}$.)

The following theorem provides a means for computing the expected length of the regenerative cycle $\bar{T}_{11}=E\left[T_{11}\right]$ in terms of the expected subcycle lengths $\vec{T}_{t j}=E\left[T_{i j}\right], i j \neq$ 11.

Theorem 1. The expected cycle length $\bar{T}_{11}(q)$ of the regenerative cycle is

$\bar{T}_{11}(q)=q+\sum_{i j \neq 11} \bar{T}_{i j} P_{11, t j}(q)$,

where $\overline{\mathbf{T}}\left[\right.$ an $(N-1) \times 1$ column vector with elements $\bar{T}_{i j}$, $i j \neq 11]$ is calculated from:

$\overline{\mathbf{T}}=(\mathbf{I}-\mathbf{Q})^{-1} \mathbf{t}$,

with $\boldsymbol{Q}$ as an $(N-1) \times(N-1)$ matrix obtained from $\boldsymbol{P}$ by removing its first column and first row and replacing the last row by $e$, and $t$ as an $(N-1) \times 1$ column vector such that $\boldsymbol{t}=[, q, q, \ldots, q, \bar{\tau}]^{\top}$ with $\bar{\tau}=E[\tau]$.

Proof. Conditioning on the state found when the inventory reaches $r$ after $q$ time units and using the renewal argument, we obtain:

$T_{11}$

$$
= \begin{cases}q, & \text { if } \left.\zeta(q)=11 \text { [with probability } P_{11,11}(q)\right], \\ q+T_{\imath}, & \text { if } \left.\zeta(q) \neq 11 \text { [with probability } P_{11, i j}(q)\right] .\end{cases}
$$

Taking expectations in (4), we have:

$\bar{T}_{11}=q+\sum_{\imath \neq 11} \bar{T}_{l j} P_{11, l j}(q)$.

We also obtain, for $i j \neq 00$,

$$
T_{i j}= \begin{cases}q, & \text { if } \zeta(q)=11 \\ q+T_{k m}, & \text { if } \zeta(q)=k m \neq 11 \\ & {\left[\text { with probability } P_{\imath \jmath, k m}(q)\right],}\end{cases}
$$

and for $i j=00$,

$T_{00}= \begin{cases}\tau+T_{10}, & {[\text { with probability } \rho],} \\ \tau+T_{01}, & {[\text { with probability } 1-\rho] .}\end{cases}$

Taking expectations in (6) and (7), simplifying, collecting the terms containing the unknowns on the left-hand side, and writing the resulting system of linear equations in terms of $\overline{\mathbf{T}}$ gives $\overline{\mathbf{T}}=\mathbf{t}+\mathbf{Q} \overline{\mathbf{T}}$, [or, $\left.\overline{\mathbf{T}}=(\mathbf{I}-\mathbf{Q})^{-1} \mathbf{t}\right]$ as claimed.

\section{b) Cycle Cost}

In order to form the expected long-run average cost function, we also need to compute the expected cost of a regenerative cycle. To this end, we define $C_{11}(q, r)$ as the random cycle cost and let $C_{l}(q, r), i j \neq 11$ be the cost of 
the subcycle that starts when the inventory drops to $r$ and the process is found in state ij. Similar to the analysis of the expected cycle length, let $\bar{C}_{i j}=E\left[C_{t j}\right], i j \in \hat{\mathscr{S}}$ and define:

$$
\begin{aligned}
\overline{\mathbf{C}}_{(N-1) \times 1}= & {\left[, \bar{C}_{12}, \ldots, \bar{C}_{10}\left|\bar{C}_{21}, \bar{C}_{22}, \ldots, \bar{C}_{20}\right|\right.} \\
& \left.\ldots \mid \bar{C}_{01}, \bar{C}_{02}, \ldots, \bar{C}_{00}\right]^{\top},
\end{aligned}
$$

as an $(N-1) \times 1$ column vector of expected subcycle lengths. Also, define $c(q, r)=K+h q^{2} / 2+h q r$ as the cost of ordering and holding inventory in an interval where inventory level starts with $q+r$ units and ends with $r$ units. (See Figure 1.) (For notational simplicity, in the subsequent discussion we will again drop the dimension index of C.)

Lemma 1. Let $\gamma(r)$ be the random cost incurred from the time inventory drops to $r$ until either supplier 1 or supplier 2 becomes available. Expected value of $\gamma(r)$ is obtained as:

$$
\begin{aligned}
\bar{\gamma}(r)=E[\gamma(r)]= & \int_{0}^{r} h\left(r w-\frac{1}{2} w^{2}\right) d A(w) \\
& +\int_{r}^{\infty}\left[\frac{1}{2} h r^{2}+b(w-r)\right] d A(w),
\end{aligned}
$$

where $A(w)$ is the limiting distribution of the forward recurrence time $\tau$ of the OFF state 00 given in (1).

Proof. First, recall that $D \equiv 1$ and $P[\tau \leqslant w]=A(w)$. Referring to Figure 1, we note that the cost incurred from the time inventory drops to $r$ until either supplier 1 or 2 become available is:

$$
\begin{aligned}
& \gamma(r) \\
& \quad= \begin{cases}h w^{2} / 2+h w(r-w)=h\left(r w-w^{2} / 2\right), & \text { if } w<r, \\
h r^{2} / 2+b(w-r), & \text { if } w \geqslant r,\end{cases}
\end{aligned}
$$

Taking expectations over $\tau$, the result follows.

Theorem 2. The expected cycle cost $\bar{C}_{11}(q, r)$ of the regenerative cycle is

$\bar{C}_{11}(q, r)=c(q, r)+\sum_{l j \neq 11} \bar{C}_{i j}(q, r) P_{11, l j}(q)$,

where $\overline{\boldsymbol{C}}$ (with elements $\bar{C}_{\imath}, i j \neq 11$ ) is calculated from

$\overline{\mathbf{C}}=(\mathbf{I}-\mathbf{Q})^{-1} \mathbf{c}$,

with $c$ as an $(N-1) \times 1$ column vector such that

$\mathbf{c}_{(N-1) \times 1}=[, c(q, r), \ldots, c(q, r), \bar{\gamma}(r)]^{\top}$.

Proof. Using conditioning arguments similar to those in Theorem 1, we have

$$
C_{11}= \begin{cases}c(q, r), & \text { if } \zeta(q)=11 \\ c(q, r)+C_{t \jmath}, & \text { if } \zeta(q) \neq 11 \\ & {\left[\text { with probability } P_{11,11}(q)\right],} \\ & \end{cases}
$$

For $i j \neq 00$,

$C_{\imath j}= \begin{cases}c(q, r), & \text { if } \zeta(q)=11 \\ & {\left[\text { with probability } P_{\imath, 11}(q)\right],} \\ c(q, r)+C_{k m}, & \text { if } \zeta(q)=k m \neq 11 \\ & {\left[\text { with probability } P_{l \jmath, k m}(q)\right],}\end{cases}$

and for $i j=00$,

$C_{00}= \begin{cases}\gamma+C_{10}, & {[\text { with probability } \rho],} \\ \gamma+C_{01}, & {[\text { with probability } 1-\rho] .}\end{cases}$

Taking expectations and using the matrix notation, we obtain $\overline{\mathbf{C}}=(\mathbf{I}-\mathbf{Q})^{-1} \mathbf{c}$ where $\mathbf{Q}$ is as defined in Theorem 1.

Now that we have expressions for the expected cycle time $\bar{T}_{11}(q)$ and expected cycle cost $\bar{C}_{11}(q, r)$, we are almost ready to form the ratio

$\mathscr{C}(q, r)=\frac{\bar{C}_{11}(q, r)}{\bar{T}_{11}(q)}$,

to compute the cost function for the problem. However, a careful look at both $\bar{T}_{11}(q)$ and $\bar{C}_{11}(q, r)$ will reveal that an exact computation of these terms requires the transient solutions of the transition functions $P_{i, k m}(q), i j, k m \in \hat{\varphi}$ for finite $q$ of the SMP of the availability process. This is presented in the next subsection.

\section{c) Transient Solution of the $\boldsymbol{P}_{i j, \mathrm{~km}}(\boldsymbol{q})$}

We will develop a system of integral equations for $P_{\imath \jmath, \mathrm{km}}(q)$ that can be solved using numerical techniques.

Recall that the ON RVs $X_{1}$ and $X_{2}$ are Erlangian with parameters $\left(s_{1}, s_{1} \lambda_{1}\right)$ and $\left(s_{2}, s_{2} \lambda_{2}\right)$, respectively. Hence, each stage of $X_{1}$ is exponential with rate $s_{1} \lambda_{1}$, i.e., it has the distribution $F_{1 t}(t)=1-e^{-s_{1} \lambda_{1} t}, t \geqslant 0, i \in \mathscr{P}_{1}$, and each stage of $X_{2}$ is exponential with rate $s_{2} \lambda_{2}$, i.e., it has the distribution $F_{2 j}(t)=1-e^{-s_{2} \lambda_{2} t}, t \geqslant 0, j \in \mathscr{S}_{2}$. The OFF RVs $Y_{1}$ and $Y_{2}$ have general distributions $G_{1}(t)$ and $G_{2}(t)$ with means $1 / \mu_{1}$ and $1 / \mu_{2}$, respectively. Define $\bar{F}_{1 i}(t)$, $\bar{F}_{2 j}(t), \bar{G}_{1}(t)$ and $\bar{G}_{2}(t)$ for $i \in \mathscr{S}_{1}$ and $j \in \mathscr{S}_{2}$ as the complementary cumulative distribution functions of the respective distributions, i.e., $\bar{F}_{1 \imath}(t)=1-F_{1 t}(t)$, etc.

To develop the transition functions (and to simplify the notation for expressing the transition functions later) it would be useful to reorder the $N$ states and list them as:

$$
\begin{aligned}
\ell_{N}^{\prime}=[ & 00,01, \ldots, 0 s_{2}\left|10,11, \ldots, 1 s_{2}\right| \\
& \left.\ldots \mid s_{1} 0, s_{1} 1, \ldots, s_{1} s_{2}\right] .
\end{aligned}
$$

Since there are $N$ states, we will have a total of $N^{2}$ transition functions $P_{l j, k m}(t), i j, k m \in \hat{\mathscr{S}}$.

Theorem 3. The transition functions $P_{y, k m}(t), t \geqslant 0$, ij, $k m \in \hat{\mathscr{S}}$ of the SMP representing the suppliers' availability status are the solutions of the following integral (renewal) equations: 


$$
\begin{aligned}
& P_{00, k m}(t) \\
& =\int_{0}^{t} d G_{1}(u) P_{10, k m}(t-u)+\int_{0}^{t} d G_{2}(u) P_{01, k m}(t-u) \\
& +\bar{G}_{1}(t) \bar{G}_{2}(t) \mathbf{1}_{\{0=k, 0=m\}}, \quad k \in \hat{\mathscr{S}}_{1}, m \in \hat{\mathscr{S}}_{2}, \\
& P_{0 j, k m}(t) \\
& =\int_{0}^{t} d G_{1}(u) P_{1 j, k m}(t-u) \\
& +\int_{0}^{t} d F_{2 \jmath}(u) P_{0(j+1), k m}(t-u)+\bar{G}_{1}(t) \bar{F}_{2 j}(t) \mathbf{1}_{\{0=k, j=m\}}, \\
& j \in \mathscr{Y}_{2}, k \in \hat{\mathscr{S}}_{1}, m \in \hat{\mathscr{S}}_{2}, \\
& P_{\imath 0, k m}(t) \\
& =\int_{0}^{t} d F_{1 \imath}(u) P_{(i+1) 0, k m}(t-u) \\
& +\int_{0}^{t} d G_{2}(u) P_{t 1, k m}(t-u)+\bar{F}_{1 l}(t) \bar{G}_{2}(t) \mathbf{1}_{\{l=k, 0=m\}}, \\
& i \in \mathscr{S}_{1}, k \in \hat{\mathscr{S}}_{1}, m \in \hat{\mathscr{S}}_{2}, \\
& P_{\imath \jmath, k m}(t) \\
& =\int_{0}^{t} d F_{1 i}(u) P_{(t+1) j, k m}(t-u) \\
& +\int_{0}^{t} d F_{2 J}(u) P_{t(J+1), k m}(t-u) \\
& +\bar{F}_{1 l}(t) \bar{F}_{2 j}(t) \mathbf{1}_{\{l=k, j=m\}}, \\
& i \in \mathscr{S}_{1}, j \in \mathscr{Y}_{2}, k \in \hat{\mathscr{S}}_{1}, m \in \hat{\mathscr{S}}_{2},
\end{aligned}
$$

with the initial conditions $P_{i, k m}(0)=1, i j=k m$, and $P_{l j, k m}(0)=0, i j \neq k m$, where $\mathbf{1}_{\{,,\}}$is the two-dimensional indicator function, and $s_{1}+1 \equiv 0$ and $s_{2}+1 \equiv 0$, for the two suppliers, respectively.

Proof. Consider (10). Condition on the first state visited at time $u$ of the first transition out of 00 . The transition may be into 10 with probability $d G_{1}(u)$, and the conditional probability of ending up in state $\mathrm{km}$ after $t-u$ time units starting at 10 is $P_{10, \mathrm{~km}}(t-u)$. The transition may also be into 01 with probability $d G_{2}(u)$ and the conditional probability of ending up in state $\mathrm{km}$ after $t-u$ time units starting at 01 is $P_{01, \mathrm{~km}}(t-u)$. Removing the conditioning and adding the probability that no transitions may have occurred by time $t$ if $0=k$ and $0=m$, that is, adding $\bar{G}_{1}(t) \bar{G}_{2}(t)$, gives the result. The other integral equations are obtained in a similar manner.

At first glance, the above system of integral Equations (10) through (13) appears to have $N^{2}$ unknown functions $P_{\imath, k m}(t)$ and $N^{2}$ equations. However, it is possible to decompose this system into a more manageable set of smaller systems of lower dimension, which substantially simplifies the solution of $P_{l \jmath, k m}(t)$.
Corollary 1. The solution of the system (10) through (13) with $N^{2}$ equations in $N^{2}$ unknown functions can be reduced to solving separately $N$ systems of $N$ integral equations in $N$ unknown functions.

Proof. Recall that $P_{i, k m}(t)$ is the conditional probability of the process being in state $\mathrm{km}$ at time $t$, given that the process started in state $i j$ at time 0 . Now, fix the index of the state to be visited at time $t$, i.e., fix $k$ and $m$ as $\bar{k}$ and $\bar{m}$, respectively. Since $s_{1}+1 \equiv 0$ and $s_{2}+1 \equiv 0$ correspond to the OFF states of the two suppliers, when $k=\bar{k}$ and $m=\bar{m}$, we can decouple (10) through (13) and reduce them to a system of $N$ integral equations in $N$ unknown functions as:

$$
\begin{aligned}
& {\left[P_{00, \bar{k} \bar{m}}(t), P_{01, \bar{k} \bar{m}}(t), \ldots, P_{0 s_{2}, \bar{k} \bar{m}}(t)\right.} \\
& \left|P_{10, \bar{k} \bar{m}}(t), P_{11, \bar{k} \bar{m}}(t), \ldots, P_{1 s_{2}, \bar{k} \bar{m}}(t)\right| \\
& \left.\quad \ldots \mid P_{s_{1} 0, \bar{k} \bar{m}}(t), P_{s_{1} 1, \bar{k} \bar{m}}(t), \ldots, P_{s_{1} s_{2}, \bar{k} \bar{m}}(t)\right] .
\end{aligned}
$$

This proves the corollary.

Remark 1. In general, even when $s_{1}$ and $s_{2}$ are small and the OFF periods are distributed exponentially, it is very difficult to solve (10) through (13) in closed form. However, as we will discuss below, numerical techniques can be used to solve the system and to compute the transient values of the transition functions $P_{\imath, k m}(t)$. In order to apply these numerical techniques effectively, it would be useful to express the individual integrals in (10) through (13) in a slightly different, but equivalent, form. For example, if we consider the first integral in (10):

$\int_{0}^{t} d G_{1}(u) P_{10, k m}(t-u)$,

we note that this is the convolution integral of two functions $g_{1}(t)$ and $P_{10, \mathrm{~km}}(t)$. By a simple change of variable, (14) can be expressed equivalently as:

$\int_{0}^{t} g_{1}(t-u) P_{10, k m}(u) d u$.

The other integrals in (10) through (13) can be rewritten in a similar manner where the transition functions and the probability densities are interchanged as in (14) and (15).

Example 1. As an example for Corollary 1, consider the case where $s_{1}=s_{2}=2$. Now, the reduced system for, e.g., $\bar{k}=\bar{m}=0$, in matrix form becomes

$\mathbf{P}_{00}(t)=\int_{0}^{t} \mathbf{H}(t-u) \mathbf{P}_{00}(u) d u+\mathbf{v}_{00}(t)$,

where

$$
\begin{aligned}
\mathbf{P}_{00}(t)= & {\left[P_{00,00}(t), P_{01,00}(t), P_{02,00}(t)\right.} \\
& \left|P_{10,00}(t), P_{11,00}(t), P_{12,00}(t)\right| \\
& \left.P_{20,00}(t), P_{21,00}(t), P_{22,00}(t)\right]^{\top},
\end{aligned}
$$


$\mathbf{H}(t) \equiv\left[\begin{array}{cccccc}0 & g_{2}(t) & 0 & g_{1}(t) & 0 & 0 \\ 0 & 0 & f_{21}(t) & 0 & g_{1}(t) & 0 \\ f_{22}(t) & 0 & 0 & 0 & 0 & g_{1}(t) \\ 0 & 0 & 0 & 0 & g_{2}(t) & 0 \\ 0 & 0 & 0 & 0 & 0 & f_{21}(t) \\ 0 & 0 & 0 & f_{22}(t) & 0 & 0 \\ f_{12}(t) & 0 & 0 & 0 & 0 & 0 \\ 0 & f_{12}(t) & 0 & 0 & 0 & 0 \\ 0 & 0 & f_{12}(t) & 0 & 0 & 0\end{array}\right.$

and, $\mathbf{v}_{00}(t) \equiv\left[\bar{G}_{1}(t) \bar{G}_{2}(t), 0,0|0,0,0| 0,0,0\right]^{\top}$. The system of integral equations in (16) can be solved numerically for the $N=9$ unknowns in $\mathbf{P}_{00}(t)$. For the other $\bar{k} \bar{m} \neq 00$, the $\mathbf{P}_{\bar{k} \bar{m}}(t)$ and $\mathbf{v}_{\bar{k} \bar{m}}(t)$ vectors assume slightly different forms, but the $\mathbf{H}(t)$ matrix does not change. In the next section we will describe a detailed example where we compute the transition functions numerically using Corollary 1 and optimize the inventory model.

\section{ANALYSIS OF TWO CASES}

In this section we describe in detail the analysis of two particular cases. The first case deals with the numerical solution of a problem with $E_{2}$ ON periods and memoryless OFF periods for both suppliers, i.e., the $E_{2}\left[E_{2}\right] / M[M]$ model. The second case considers the problem where the optimal $q$ may be "large," hence we can approximate the transient solution of the transition functions of the SMP by their limiting values.

\subsection{Numerical Solution of the $E_{2}\left[E_{2}\right] / M[M]$ Model}

In this case $s_{1}=s_{2}=2$, i.e., both $X_{1}$ and $X_{2}$ are Erlangian with parameters $\left(2,2 \lambda_{1}\right)$ and $\left(2,2 \lambda_{2}\right)$, respectively. This implies that $F_{1 i}(t)=1-e^{-2 \lambda_{1} t}, t \geqslant 0, i=1,2$, and $F_{2 j}(t)$ $=1-e^{-2 \lambda_{2} t}, t \geqslant 0, j=1,2$. For simplicity, we assume that the OFF RVs $Y_{1}$ and $Y_{2}$ are exponential with parameters $\mu_{1}$ and $\mu_{2}$, i.e., $G_{1}(t)=1-e^{-\mu_{1} t}$ and $G_{2}(t)=1-$ $e^{-\mu_{2} t}, t \geqslant 0$. We have $\mathscr{S}_{1}=\{1,2\}$ and $\mathscr{S}_{2}=\{1,2\}$; hence, there are a total of $N=(2+1)(2+1)=9$ states listed as $\ell_{N}^{\prime}=[00,01,02|10,11,12| 20,21,22]$.

In order to compute the expected cost $\mathscr{C}(q, r)=\bar{C}_{11}(q$, $r) / \bar{T}_{11}(q)$, we need the transient solution of the transition functions $P_{l l, k m}(q)$ for each $q \geqslant 0$. We now describe the numerical evaluation of the transition functions.

As we discussed in Corollary 1 and demonstrated in Example 1 , for fixed $\bar{k}$ and $\bar{m}$, say, $\bar{k}=\bar{m}=0$, we would have to solve a system of nine integral Equations (16) in the nine unknown functions (17). Since the unknown functions appear inside the integrals in (16), solution of the integral equations would have to be performed using numerical integration techniques. The integral equations in (16) are classified as "Volterra type of the second kind"
GÜRLER AND PARLAR /

$\left.\begin{array}{ccc}0 & 0 & 0 \\ 0 & 0 & 0 \\ 0 & 0 & 0 \\ f_{11}(t) & 0 & 0 \\ 0 & f_{11}(t) & 0 \\ 0 & 0 & f_{11}(t) \\ 0 & g_{2}(t) & \\ 0 & 0 & f_{21}(t) \\ f_{22}(t) & 0 & 0\end{array}\right]$,

(Jerri 1985). There are several numerical solution methods that can be used to compute the integrals in (16). These include successive approximations (Jerri 1985), Laplace transforms (Bellman et al. 1966), and direct numerical integration (Jerri 1985). We have tried all three methods and decided to use the last one for its numerical stability.

The direct method of solving integral equations is based on approximating an integral using one of many classical formulas for equally spaced points in the domain of integration. These include, for example, trapezoidal rule, Simpson's rule, and Bode's rule (Press et al. 1989). Briefly stated, for a scalar integral equation such as:

$P(t)=\bar{G}(t)+\int_{0}^{t} g(t, u) P(u) d u, \quad P(0)=\bar{G}(0)=1$,

for given $\bar{G}(t)$ and $g(t, u) \equiv g(t-u)$ with the unknown function $P(t), t \geqslant 0$, one approximates the integral, say, using the trapezoidal rule, as:

$$
\begin{aligned}
& \int_{0}^{t} g(t, u) P(u) d u \\
& \cong \Delta t\left[\frac{1}{2} g\left(t, u_{1}\right) P\left(u_{1}\right)+g\left(t, u_{2}\right) P\left(u_{2}\right)+\cdots\right. \\
& \left.\quad+g\left(t, u_{n-1}\right) P\left(u_{n-1}\right)+\frac{1}{2} g\left(t, u_{n}\right) P\left(u_{n}\right)\right],
\end{aligned}
$$

where the interval of integration $[0, t]$ is divided into $n$ equal subintervals of length $\Delta t=t / n ; u_{j} \leqslant t, j \geqslant 1 ; u_{1}=0$ and $u_{n}=t$. (The integration is over $u, 0 \leqslant u \leqslant t$; thus, for $u>t, g(t, u)=0$.)

The integral equation in (19) can now be approximated by the sum:

$$
\begin{aligned}
& P(t) \\
& \qquad \bar{G}(t)+\Delta t\left[\frac{1}{2} g\left(t, u_{1}\right) P\left(u_{1}\right)+g\left(t, u_{2}\right) P\left(u_{2}\right)+\cdots\right. \\
& + \\
& \quad+g\left(t, u_{n-1}\right) P\left(u_{n-1}\right) \\
& \left.+\frac{1}{2} g\left(t, u_{n}\right) P\left(u_{n}\right)\right] .
\end{aligned}
$$

If we consider $n$ sample values of $P(t), P\left(u_{t}\right)=P_{l}, i=$ $1, \ldots, n$, Equation (21) becomes a system of $n$ linear equations in $n$ unknowns $P_{l}$, i.e., 


$$
\begin{aligned}
& P_{1}=\bar{G}_{1}, \\
& P_{\imath}=\bar{G}_{\imath} \\
& +\Delta t\left[\frac{1}{2} g_{t 1} P_{1}+g_{\imath 2} P_{2}+\cdots+g_{t, n-1} P_{n-1}+\frac{1}{2} g_{l n} P_{n}\right], \\
& \quad i=2, \ldots, n,
\end{aligned}
$$

where $\bar{G}_{\imath}=\bar{G}\left(u_{i}\right), g_{\imath j}=g\left(t_{i}, u_{j}\right), j \leqslant i$, and $u_{j} \leqslant t_{t}$. Hence the numerical solution of an integral equation can be reduced to solving a system of $n$ linear equations in $n$ unknowns. This linear system can be conveniently expressed as $\tilde{\mathbf{P}}=\tilde{\mathbf{H}} \tilde{\mathbf{P}}+\overline{\mathbf{v}}$, with the solution $\tilde{\mathbf{P}}=(\mathbf{I}-\tilde{\mathbf{H}})^{-1} \tilde{\mathbf{v}}$, where $\tilde{\mathbf{v}}=\left[\bar{G}_{1}, \ldots, \bar{G}_{n}\right]^{\top}$ and $\tilde{\mathbf{P}}=\left[P_{1}, \ldots, P_{n}\right]^{\top}$ are $n \times 1$ column vectors and:

$$
\tilde{\mathbf{H}}=\Delta t\left[\begin{array}{ccccc}
0 & 0 & \cdots & 0 & 0 \\
\frac{1}{2} g_{21} & \frac{1}{2} g_{22} & \cdots & 0 & 0 \\
\vdots & \vdots & \ddots & \vdots & \vdots \\
\frac{1}{2} g_{n 1} & g_{n 2} & \cdots & g_{n, n-1} & \frac{1}{2} g_{n n}
\end{array}\right]
$$

is an $n \times n$ matrix.

Of course, our problem is complicated by the fact that we have a system of integral equations in $N$ unknown functions $P_{i j, k m}(t), t \geqslant 0$, for fixed $\bar{k}$ and $\bar{m}$. However, we can still apply the trapezoidal rule to each integral in (16) after dividing the $[0, t]$ interval into $n$ subintervals of equal length. Since there are $N$ unknown functions, each of which is being sampled at $n$ points, the resulting linear system now has $n N$ equations in $n N$ unknowns. For example, for $\bar{k}=\bar{m}=0$, this would transform (16) into:

$\hat{\mathbf{P}}_{00}=\hat{\mathbf{H}} \hat{\mathbf{P}}_{00}+\hat{\mathbf{v}}_{00}$,

where

$$
\begin{aligned}
\hat{\mathbf{P}}_{00}=[ & P_{00}\left(u_{1}\right), \ldots, P_{00}\left(u_{n}\right)\left|P_{01}\left(u_{1}\right), \ldots, P_{01}\left(u_{n}\right)\right| \\
& \left.\ldots \mid P_{s_{1} s_{2}}\left(u_{1}\right), \ldots, P_{s_{1} s_{2}}\left(u_{n}\right)\right], \\
\hat{\mathbf{v}}_{00}=[ & \bar{G}_{1}\left(u_{1}\right) \bar{G}_{2}\left(u_{1}\right), \ldots, \bar{G}_{1}\left(u_{n}\right) \bar{G}_{2}\left(u_{n}\right) \\
& |0, \ldots, 0| \ldots \mid 0, \ldots, 0]
\end{aligned}
$$

are $n N$ dimensional column vectors and $\hat{\mathbf{H}}$ is a suitably constructed sparse $n N \times n N$ matrix whose nonzero submatrices are positioned in a manner similar to the nonzero entries in (18).

Solving (22) for $\hat{\mathbf{P}}_{00}$ would give the numerically estimated solution of the transition functions $P_{i j, 00}(t)$ for any $t \geqslant 0$. The computational approach is very similar for other $\bar{k} \bar{m} \neq 00$; however, $\hat{\mathbf{v}}_{\bar{k} \bar{m}}$ assume slightly different forms.

Now that we have estimates for all the transition functions $P_{i l, k m}(t), i j, k m \in \mathscr{S}$, we can easily compute $\overline{\mathbf{T}}$ in (3) and find the expected cycle time $\bar{T}_{11}(q)$ for any given $q$. Similarly, $\overline{\mathbf{C}}$ can be calculated from (9) and the expected cycle cost $\vec{C}_{11}(q, r)$ can be found for given $q$ and $r$ values. Using $\bar{T}_{11}(q)$ and $\bar{C}_{11}(q, r)$, one can then find the corresponding value of the expected cycle cost $\mathscr{C}(q, r)$ for given $q$ and $r$.

Unfortunately, since neither $\bar{T}_{11}(q)$ nor $\bar{C}_{11}(q, r)$ is available in closed form, it is not possible to analyze $\mathscr{C}(q, r)$ for its convexity properties nor to compute its partial derivatives. Therefore, it would be useful to implement a search method that can identify the optimum making use of different values of $\mathscr{C}$ evaluated at $(q, r)$. We have decided to optimize $\mathscr{C}(q, r)$ using adaptive random search (ARS) (Gottfried and Weisman 1973) for its simplicity and effectiveness. ARS is a simpler version of the global optimization method known as simulated annealing (van Laarhoven and Aarts 1987). For our problem with two decision variables, starting with a feasible point in the twodimensional region that is known to contain the optimal solution $\left(q^{*}, r^{*}\right)$, ARS randomly generates the next feasible point and compares it to the previous point. If the new point is better than the previous one, it is kept; otherwise, the new point is discarded. For example, if $q^{*}$ is known to be in the interval $\left[q_{\ell}, q_{\ell}\right.$ ], and if $q_{\text {old }}$ is the previous point, then the new point $q_{\text {new }}$ is generated using the formula $q_{\text {new }}=q_{\text {old }}+\left(q_{\hbar}-q_{\ell}\right)(2 \theta-1)^{\nu}$, where $\theta$ is a random number between 0 and 1 and $v$ is an odd integer. The process continues in this manner until convergence is established.

We use the following algorithm to compute the optimal $(q, r)$ values:

Step 0. Set the initial value of $\mathscr{C}$ at a high positive level, say, $10^{6}$.

Step 1. Start with a feasible $(q, r)$ point.

Step 2. Evaluate the transition functions at $q$ using the method that solves the system of integral equations for $P_{\imath, k m}(q)$.

Step 3. Generate the $\mathbf{Q}$ matrix appearing in (3). Compute the $\overline{\mathbf{T}}$ and $\overline{\mathbf{C}}$ vectors and evaluate $\bar{T}_{11}(q)$ and $\bar{C}_{11}(q$, $r)$.

Step 4. Evaluate the expected cost $\mathscr{C}(q, r)$. If the new value of $\mathscr{C}$ is better than the previous one, keep the corresponding $(q, r)$. (If the improvements in $\mathscr{C}$ become negligible, stop.) Go to Step 2. Otherwise, generate a new feasible $(q, r)$ and go to Step 2.

Example 2. Let us now consider an example where the above algorithm is implemented. Supplier availability and cost parameters are initially set as follows: $\lambda_{1}=3 / 5, \lambda_{2}=$ $1 / 2, \mu_{1}=1, \mu_{2}=3 / 4 ; K=\$ 200 /$ order, $h=\$ 100 /$ unit $/$ time, $b=\$ 500 /$ unit. As previously discussed, demand rate is $D \equiv 1$ unit/time where a "unit" could correspond to, say, 1,000 items. Since $Y_{1}$ and $Y_{2}$ are exponential, it can easily be shown that $A(w)=1-e^{-\mu w}, w \geqslant 0, \rho=\mu_{1} / \mu, \bar{\tau}=$ $1 / \mu$, and $\bar{\gamma}(r)=e^{-\mu r}\left[h e^{\mu r}(\mu r-1)+b \mu+h\right] / \mu^{2}$, where $\mu=\mu_{1}+\mu_{2}$.

To implement the ARS optimization algorithm, we wrote a program using Microsoft QuickBASIC v4.5 which was run on an 80486 machine with a clock speed of 50 MHz. Numerical solution of the integral equations (16) was performed using a value of $n=10$. This meant that, to solve for $\hat{\mathbf{P}}_{00}$ in (22), we had to compute the inverse of a 
Table I

Sensitivity Analysis Results for $E_{2}\left[E_{2}\right] / M[M]$ Model

\begin{tabular}{|c|c|c|c|c|c|c|c|}
\hline $\begin{array}{c}K \\
(1)\end{array}$ & $\begin{array}{c}h \\
(2)\end{array}$ & $\begin{array}{c}b \\
(3)\end{array}$ & $\begin{array}{l}q^{*} \\
(4)\end{array}$ & $\begin{array}{l}r^{*} \\
(5)\end{array}$ & $\begin{array}{l}\mathscr{C}^{*} \\
(6)\end{array}$ & $\begin{array}{c}q_{\mathrm{EOO}}^{*} \\
(7)\end{array}$ & $\begin{array}{c}\mathscr{C}_{\text {EOQ }}^{*} \\
(8)\end{array}$ \\
\hline \multirow[t]{4}{*}{200} & 100 & 500 & 2.119 & 0.004 & 211.63 & 2.000 & 200.00 \\
\hline & & 1,000 & 2.239 & 0.018 & 228.15 & & \\
\hline & 300 & 500 & 1.170 & 0.000 & 354.91 & 1.154 & 346.41 \\
\hline & & 1,000 & 1.208 & 0.008 & 382.99 & & \\
\hline \multirow[t]{4}{*}{400} & 100 & 500 & 2.952 & 0.000 & 289.19 & 2.828 & 282.84 \\
\hline & & 1,000 & 3.016 & 0.002 & 303.04 & & \\
\hline & 300 & 500 & 1.731 & 0.003 & 492.08 & 1.632 & 489.89 \\
\hline & & 1,000 & 1.814 & 0.012 & 516.41 & & \\
\hline
\end{tabular}

$90 \times 90$ matrix I $-\hat{\mathbf{H}}$. The bottleneck in these computations is the size of $\mathbf{I}-\hat{\mathbf{H}}$ which would quickly grow as $s_{1}$ and/or $s_{2}$ take on larger values.

In all computations we used double precision arithmetic. Inversion of the $90 \times 90$ matrix using LU decomposition was done only once, which took approximately one minute. Generation of the $\mathbf{Q}$ matrix and the evaluation of the $\mathscr{E}(q$, $r)$ function had to be done for each test value of $(q, r)$ used in the algorithm. Each iteration took about 4 seconds. Using the above data values, we obtained the optimal values as $q^{*}=2.119$ unit, $r^{*}=0.004$ unit, and $\mathscr{C}^{*}=\$ 211.63$. In the absence of supplier uncertainties, we would use the standard EOQ model for which the optimal solution would be $q_{\mathrm{EOQ}}^{*}=2.0, r_{\mathrm{EOQ}}^{*}=0$, and $\mathscr{C}_{\mathrm{EOQ}}^{*}=\$ 200.00$, implying that the cost of uncertainty is $\$ 11.63$ per time unit.

We have solved a large number (several hundred) of other test problems to evaluate the effects of changing parameter values on the optimal solution. In Table I we present a representative sample of eight problems that indicate the direction of movements for $q^{*}, r^{*}$, and $\mathfrak{C}^{*}$ given in Columns 4 to 6 when $K, h$, and $b$ vary as in Columns 1 to 3 . This table includes the corresponding values for the optimal solution for the standard EOQ model as given in Columns 7 and 8 . Note that optimal order quantity $q^{*}$ in the presence of supply disruptions is always greater than the economic order quantity $q_{\mathrm{EOQ}}^{*}$. Although the optimal reorder point $r^{*}$ is not kept at very high levels, it is affected by increases in the backorder cost $b$, as should be expected.

\subsection{Analysis of the Model for "Large" $q$}

In this subsection we analyze the model for asymptotically "large" values of $q$ so that the transient probabilities can simply be replaced by their limiting values $P_{k m}=\lim _{q \rightarrow \infty}$ $P\{\zeta(q)=k m \mid \zeta(0)=i j\}, k m \in \hat{9}$ in order to simplify the analysis. When optimal $q$ is not likely to be large (in relation to $D \equiv 1$ ), then this approach would provide an easy to compute, but possibly poor approximate solution. On the other hand, if order cost $K$ is large and/or holding cost $h$ is small compared to backorder cost $b$, then the optimal $q$ is likely to be large, and replacing the above time-dependent (transient) conditional probabilities by their constant limiting values may be an acceptable approximation. (This will be more apparent in Example 3 that will be discussed later in this section.)

\section{a) Computation of the Limiting Probabilities}

Analysis of the model for large $q$ produces the limiting probabilities of the SMP of the $N$ states in the problem. Since $N$ is finite and $\mathrm{ON}$ and OFF periods of both suppliers have finite means, the SMP has a unique limiting distribution independent of the initial state (Ross 1983, p. 131). Under these conditions, we have the following proposition.

Proposition 1. The limiting probabilities of the SMP:

$P_{k m}=\lim _{q \rightarrow \infty} P\{\zeta(q)=k m \mid \zeta(0)=i j\}, i j, k m \in \hat{\mathscr{S}}$,

exist independent of the initial state, and they are computed as:

$$
\begin{aligned}
& P_{00}=\frac{E\left(Y_{1}\right)}{1 / \lambda_{1}+E\left(Y_{1}\right)} \cdot \frac{E\left(Y_{2}\right)}{1 / \lambda_{2}+E\left(Y_{2}\right)}, \\
& P_{0 m}=\frac{E\left(Y_{1}\right)}{1 / \lambda_{1}+E\left(Y_{1}\right)} \cdot \frac{1 /\left(s_{2} \lambda_{2}\right)}{1 / \lambda_{2}+E\left(Y_{2}\right)}, \quad m \in \mathscr{S}_{2}, \\
& P_{k 0}=\frac{1 /\left(s_{1} \lambda_{1}\right)}{1 / \lambda_{1}+E\left(Y_{1}\right)} \cdot \frac{E\left(Y_{2}\right)}{1 / \lambda_{2}+E\left(Y_{2}\right)}, \quad k \in \mathscr{S}_{1}, \\
& P_{k m} \\
& =\frac{1 /\left(s_{1} \lambda_{1}\right)}{1 / \lambda_{1}+E\left(Y_{1}\right)} \cdot \frac{1 /\left(s_{2} \lambda_{2}\right)}{1 / \lambda_{2}+E\left(Y_{2}\right)}, \quad k \in \mathscr{S}_{1}, m \in \mathscr{Y}_{2} .
\end{aligned}
$$

Proof. First recall that the two suppliers have independent ON/OFF processes $\zeta_{1}=\left\{\zeta_{1}(t), t \geqslant 0\right\}$ and $\zeta_{2}=\left\{\zeta_{2}(t), t \geqslant\right.$ $0\}$. Consider the limiting probabilities of $\zeta_{1}$ and $\zeta_{2}$, i.e.,

$P_{k}^{(1)}=\lim _{q \rightarrow \infty} P\left\{\zeta_{1}(q)=k \mid \zeta_{1}(0)=i\right\}, \quad i, k \in \hat{\mathscr{Y}}_{1}$, $P_{m}^{(2)}=\lim _{q \rightarrow \infty} P\left\{\zeta_{2}(q)=m \mid \zeta_{2}(0)=j\right\}, \quad j, m \in \hat{\mathscr{Y}}_{2}$.

Since the sum of the $\mathrm{ON}$ and OFF periods have finite means for both suppliers, i.e., $E\left(X_{1}+Y_{1}\right)<\infty$ and $E\left(X_{2}\right.$ $\left.+Y_{2}\right)<\infty$, using Proposition 4.8.1 in Ross (1983, p. 131), we obtain:

$$
\begin{aligned}
P_{k}^{(1)} & =\frac{1 /\left(s_{1} \lambda_{1}\right)}{1 / \lambda_{1}+E\left(Y_{1}\right)}, \quad k \in \mathscr{Y}_{1}, \\
P_{0}^{(1)} & =\frac{E\left(Y_{1}\right)}{1 / \lambda_{1}+E\left(Y_{1}\right)}, \\
P_{m}^{(2)} & =\frac{1 /\left(s_{2} \lambda_{2}\right)}{1 / \lambda_{2}+E\left(Y_{2}\right)}, \quad m \in \mathscr{Y}_{2}, \\
P_{0}^{(2)} & =\frac{E\left(Y_{2}\right)}{1 / \lambda_{2}+E\left(Y_{2}\right)},
\end{aligned}
$$

as the limiting probabilities of the ON/OFF processes for the individual suppliers. Using the independence of the $\zeta_{1}$ and $\zeta_{2}$ processes, we have:

$$
\begin{aligned}
& P_{00}=P_{0}^{(1)} P_{0}^{(2)}, \\
& P_{0 m}=P_{0}^{(1)} P_{m}^{(2)}, \quad m \in \mathscr{S}_{2}, \\
& P_{k 0}=P_{k}^{(1)} P_{0}^{(2)}, \quad k \in \mathscr{S}_{1}, \\
& P_{k m}=P_{k}^{(1)} P_{m}^{(2)}, \quad k \in \mathscr{S}_{1}, m \in \mathscr{S}_{2} .
\end{aligned}
$$




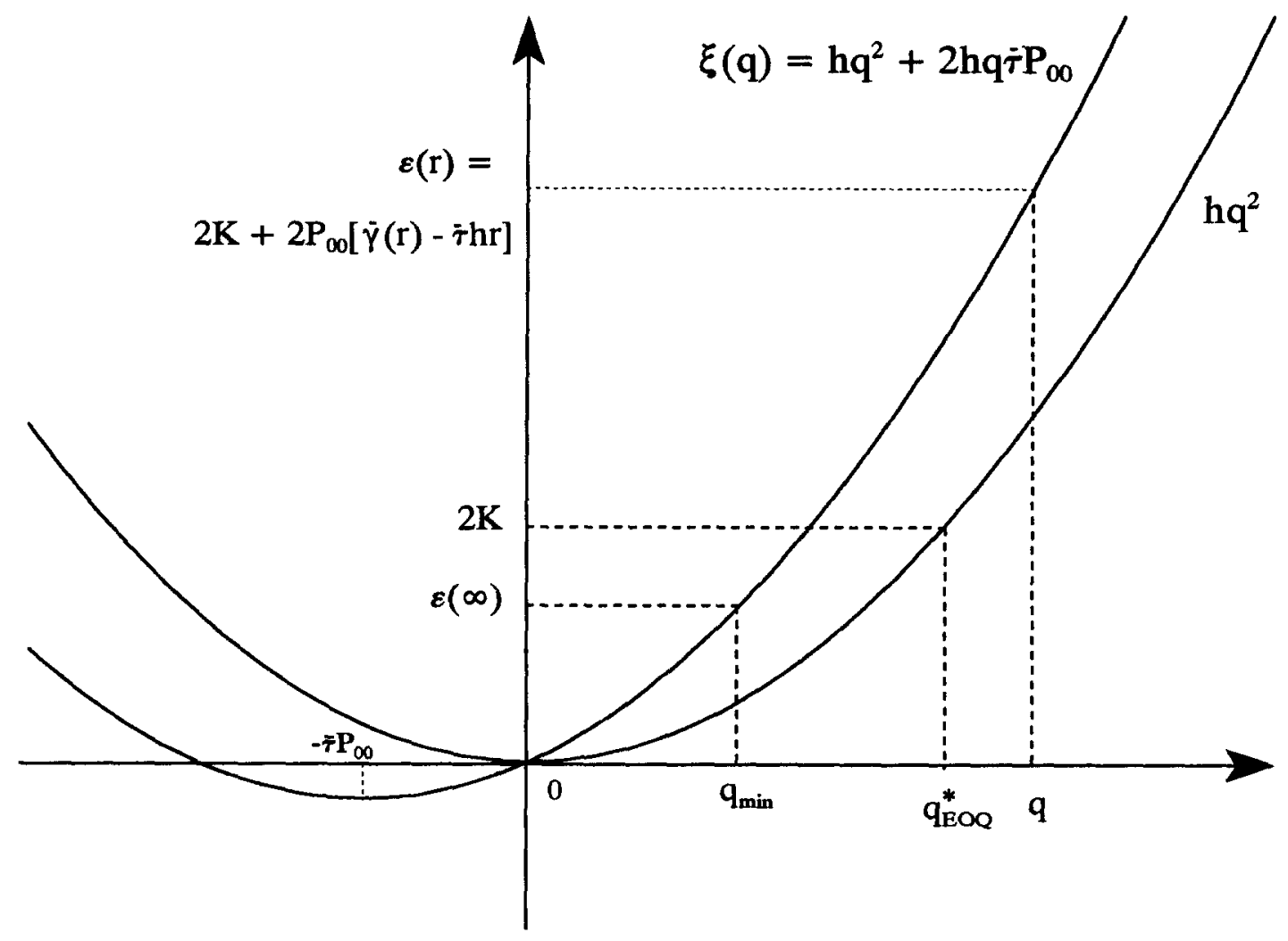

Figure 3. Analysis of $\mathscr{K}_{q}(q, r)=0$.

\section{b) The Cost Function}

The following theorem provides simple expressions for the expected cycle length and cycle cost (and hence the expected cost) when $q$ is "large."

Theorem 4. Assume that the limiting probabilities $P_{k m}$ of the SMP have been found using the procedure described above. When $q$ is "large," the long-run expected average cost function $\mathscr{K}(q, r)$ is obtained as:

$\mathscr{K}(q, r)=\frac{c(q, r)+\bar{\gamma}(r) P_{00}}{q+\bar{\tau} P_{00}}$.

Proof. We find (after some matrix manipulations) that:

$$
\begin{gathered}
\overline{\mathbf{T}}=(\mathbf{I}-\mathbf{Q})^{-1} \mathbf{t}=\frac{1}{P_{11}}\left[P_{00} \bar{\tau}+q, \ldots, P_{00} \bar{\tau}+q,\right. \\
\left.q+\bar{\tau}\left(P_{00}+P_{11}\right)\right]^{\top},
\end{gathered}
$$

i.e., the first $N-2$ elements of the $\overline{\mathbf{T}}$ vector are $\left(P_{00} \bar{\tau}+\right.$ $q) / P_{11}$, and the last element is $\bar{\tau}\left(P_{00}+P_{11}\right) / P_{11}$. Using $\bar{T}_{11}=q+\Sigma_{i j \neq 11} \bar{T}_{i j} P_{l y}$, we obtain the expression for the expected cycle time as $\bar{T}_{11}(q)=\left(q+\bar{\tau} P_{00}\right) / P_{11}$. Similarly, for the expected cycle length we find another expression as $\bar{C}_{11}(q, r)=\left[c(q, r)+\bar{\gamma}(r) P_{00}\right] / P_{11}$. Using the renewal reward theorem, the objective function becomes:

$\mathscr{K}(q, r)=\frac{\bar{C}_{11}(q, r)}{\bar{T}_{11}(q)}=\frac{c(q, r)+\bar{\gamma}(r) P_{00}}{q+\bar{\tau} P_{00}}$.
When both suppliers are always available, then $P_{00}=0$ and the above expression reduces to the cost function of the standard EOQ model, as would be expected.

It is interesting to note that, when $q$ is large the only probability that appears in the objective function is $P_{00}$, i.e., the limiting probability of finding both suppliers in their OFF state. This is the case when shortages may start accumulating, and the cycle time and cycle cost differ from those of the EOQ model, i.e., from $q$ and $c(q, r)$ by an amount of $\bar{\tau}$ and $\bar{\gamma}(r)$, respectively.

\section{c) Optimality Conditions}

We now analyze the cost function (23) in more detail by examining the necessary conditions for optimality. (In what follows, we use the subscript notation to denote the partial derivatives of $\mathscr{K}(q, r)$ and $c(q, r)$.)

(i) $\mathscr{K}_{q}(q, r)=0$ implies $\left(q+\bar{\tau} P_{00}\right) c_{q}(q, r)-c(q, r)=$ $\bar{\gamma}(r) P_{00}$, which simplifies to $\xi(q) \equiv h q^{2}+2 h q \bar{\tau} P_{00}=\epsilon(r)$ $\equiv 2 K+2 P_{00}[\bar{\gamma}(r)-\bar{\tau} h r]$. Here, $\xi(q)$ is a convex quadratic function with $\xi(0)=0$, and it has a minimum at $q=-\bar{\tau} P_{00}$ such that $\xi\left(-\bar{\tau} P_{00}\right)=-h \bar{\tau}^{2} P_{00}^{2}$. For a given value of $r, \epsilon(r)$ is a constant, and the corresponding value of $q$ can be computed by solving the quadratic equation $\xi(q)=\epsilon(r)$. (See Figure 3.) Also, note that if $P_{00}=0$, i.e., when the suppliers are always available, then $\xi(q)=h q^{2}=\epsilon(r)=$ $2 K$ so that the optimal order quantity is $q^{*}=q_{\mathrm{EOO}}^{*}=$ $\sqrt{2 K / h}$. This is the solution for the classical EOQ problem with demand $D \equiv 1$, as expected. 
GÜRLER AND PARLAR

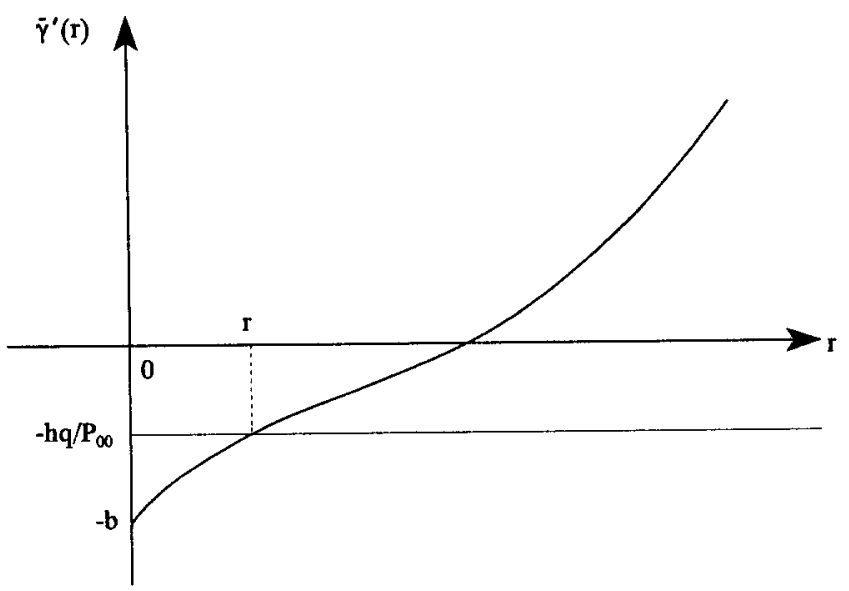

Figure 4. Analysis of $\mathscr{K}_{r}(q, r)=0$.

For positive $P_{00}$, the smallest value of $[\bar{\gamma}(r)-\bar{\tau} h r]$ (obtained as $r \rightarrow \infty)$ is $-h E\left(\tau^{2}\right) / 2$, hence solving $\xi(q)=\epsilon(\infty)$ $=2 K-h E\left(\tau^{2}\right) / 2$ gives the lower bound $q_{\min }=\xi^{-1}[2 K-$ $\left.h E\left(\tau^{2}\right) / 2\right]$ for the optimal order quantity $q^{*}$, i.e., $q_{\min } \leqslant q^{*}$.

(ii) $\mathscr{K}_{r}(q, r)=0$ implies $c_{r}(q, r)+P_{00} \bar{\gamma}^{\prime}(r)=0$, which simplifies to:

$\bar{\gamma}^{\prime}(r)=-h q / P_{00}$

where $\bar{\gamma}^{\prime}(r)=h \int_{0}^{r} w d A(w)+(h r-b) \int_{r}^{\infty} d A(w)$. Differentiating $\bar{\gamma}^{\prime}(r)$ once more, we obtain $\bar{\gamma}^{\prime \prime}(r)=h \int_{r}^{\infty} d A(w)+$ $b A^{\prime}(r)$, which is always positive, implying that $\bar{\gamma}(r)$ is convex and $\bar{\gamma}^{\prime}(r)$ is an increasing function of $r$. (See Figure 4.) Note that $\bar{\gamma}^{\prime}(0)=-b$, and if $-b<-h q P_{00}$ for a given $q$, then there exists a unique value of $r$ solving (24), since $\bar{\gamma}^{\prime}(r)$ was shown to be increasing in $r$. If $-h q P_{00}<-b$ for some $q$, then $r$ would be set equal to zero.

The above system $\mathscr{K}_{q}=\mathscr{K}_{r}=0$ could be solved iteratively as in the continuous-review $(q, r)$ model discussed in Hadley and Whitin (1963, Section 4.4). One would start with $q=q_{\mathrm{EOQ}}^{*}=\sqrt{2 K / h}$ and solve for $r$ in $\mathscr{K}_{r}=0$. This value of $r$ would be used to compute a new $q$ from $\mathscr{K}_{q}=0$ until convergence is achieved.

Using the necessary conditions provided above, one can show that $\mathscr{K}_{r r}>0$ and the determinant $|\mathscr{H}|$ of the Hessian matrix

$\mathscr{H}=\left[\begin{array}{ll}\mathscr{K}_{q q} & \mathscr{H}_{q r} \\ \mathscr{K}_{r q}^{q} & \mathscr{K}_{r r}\end{array}\right]$,

is found as

$$
\begin{aligned}
|\mathscr{H}|\left(P_{00} \bar{\tau}+q\right)^{4} / P_{00} & \\
= & \alpha(r) \equiv \bar{\gamma}^{\prime \prime}(r)\left[2 P_{00} \bar{\gamma}(r)+2 K+P_{00} h \bar{\tau}\left(P_{00} \bar{\tau}-2 r\right)\right] \\
& -P_{00}\left[\bar{\gamma}^{\prime}(r)-h \bar{\tau}\right]^{2} .
\end{aligned}
$$

Since $\alpha(r)$ is a function of $r$ only, for given parameter values its sign can be easily checked for positiveness, i.e., for the convexity of the function $\mathscr{K}(q, r)$. If $\mathscr{K}(q, r)$ is everywhere convex, then the iterative method suggested above would converge to the optimal solution. As $K$ is likely to be "large," since $q$ was assumed "large," $\bar{\gamma}(r)>$
Table II

Efficiency of the Asymptotic Approximation

\begin{tabular}{ccrccccc}
\hline$K$ & $h$ & \multicolumn{1}{c}{$b$} & $\bar{q}$ & $\bar{r}$ & $\mathscr{C}(\bar{q}, \bar{r})$ & $\mathscr{C}^{*}$ & $\mathscr{C}(\bar{q}, \bar{r}) / \mathscr{C}^{*}$ \\
$(1)$ & $(2)$ & $(3)$ & $(4)$ & $(5)$ & $(\overline{6})$ & $(7)$ & $(8)$ \\
\hline 200 & 100 & 500 & 2.17 & 0.013 & 212.34 & 211.63 & 1.003 \\
& & 1,000 & 2.35 & 0.004 & 228.60 & 228.15 & 1.002 \\
& 300 & 500 & 1.34 & 0.084 & 378.36 & 354.91 & 1.066 \\
& & 1,000 & 1.45 & 0.000 & 386.38 & 382.99 & 1.008 \\
400 & 100 & 500 & 2.93 & 0.090 & 295.82 & 289.19 & 1.022 \\
& & 1,000 & 3.08 & 0.028 & 304.49 & 303.04 & 1.004 \\
& 300 & 500 & 1.77 & 0.058 & 506.51 & 492.08 & 1.029 \\
& & 1,000 & 1.87 & 0.107 & 538.89 & 516.41 & 1.043 \\
\hline
\end{tabular}

0 , and $0<P_{00}<1, \alpha(r)$ would likely be a positive function of $r$, hence $\mathscr{K}(q, r)$ would be convex.

Example 3. We used the supplier availability parameters of Example 2 and the cost data in Table I to compute the "optimal" solution $\bar{q}$ and $\bar{r}$ that minimize $\mathscr{K}(q, r)$. The results are presented in Table II, where we compare the expected cost of the exact version of the cost function $\mathscr{C}$ evaluated at $(\bar{q}, \bar{r})$ with the minimum expected cost $\ell^{*}$ found in Example 2. As we had indicated at the start of this section, when $K$ is large and/or $h$ is small compared to $b$, the asymptotic approximation is likely to give rise to very accurate results. In fact, as we see in Table II, for $b=1,000$ and "large" $\bar{q}>2$, we obtain the smallest error in the solution, where $\mathscr{C}(\bar{q}, \bar{r})$ exceeds $\mathscr{b}^{*}$ by less than $0.8 \%$. When the conditions for $K$ and $b$ (as specified above) are satisfied for a given problem, it is advisable to use the asymptotic approximation of this section to compute the optimal values of decision variables.

\section{ANALYSIS OF AN ALTERNATIVE INVENTORY POLICY}

When at least one of the suppliers is always available, the optimal policy would call for ordering $q^{*}=\sqrt{2 K / h}$ units whenever the inventory level drops to the reorder point of $r^{*}=0$, i.e., the standard EOQ model applies. In the general environment with two suppliers who may be independently ON or OFF for random lengths of time, one can use one of many different inventory policies. One is the relatively simple to implement $(q, r)$ policy developed in this paper that requires the optimization of a cost function of two variables. Another policy is to make the order decisions dependent on the number of available suppliers. In this case, there would be two different order quantities represented by the vector $\mathbf{q}^{(2)}=\left(q_{1}, q_{2}\right)$, where $q_{J}$ is the order quantity when the number of available suppliers is $j$, $j=1,2$. The resulting $\left(\mathbf{q}^{(2)}, r\right)$ policy could be modeled in a similar manner. However, the expressions for cycle length and cycle cost would be different, and the objective function would depend on three decision variables. It is clear that this policy is more general than the $(q, r)$ policy. This follows, since if $q_{1}=q_{2}$, then $\left(\mathbf{q}^{(2)}, r\right)$ reduces to $(q$, $r)$. 
Yet another possibility is to make the decisions "statedependent" in the sense that the order quantity would depend on the availability status of the suppliers. Now, one would need to consider three different order quantities represented by the vector $\mathbf{q}^{(3)}=\left(q_{11}, q_{10}, q_{01}\right)$ and the reorder point $r$. Here, $q_{11}$ is the quantity ordered when both suppliers are $\mathrm{ON}, q_{10}$ is the quantity ordered when only supplier 1 is $\mathrm{ON}$, and $q_{01}$ is the quantity ordered if only supplier 2 is ON when inventory level drops to $r$. This $\left(\mathbf{q}^{(3)}, r\right)$ policy is even more general than $\left(\mathbf{q}^{(2)}, r\right)$, since setting $q_{01}=q_{10}$ would reduce $\left(\mathbf{q}^{(3)}, r\right)$ to $\left(\mathbf{q}^{(2)}, r\right)$.

In the model that uses the $\left(q^{(3)}, r\right)$ policy, it may be tempting to define the cycles to commence whenever the inventory is raised to $q_{11}+r$ when $\zeta(t)=11$ and proceed as in Section 1. However, this would not be an operational definition of a cycle, since we do not actually observe state 11 of the process as the stages of Erlang random variables are artificial constructs. (For the simple $(q, r)$ policy of Section 1, this was not a problem since the order quantity was not state dependent, and we always ordered $q$ units regardless of which supplier was available.) Thus, in the $\left(\mathbf{q}^{(3)}, r\right)$ model one would need to redefine the cycles to start whenever the inventory level is raised to $q_{10}+r$ units at that epoch where supplier 1 just commences his ON period and supplier 2 is still OFF. (Equivalently, cycles may also be defined to start when inventory is raised to $q_{01}+r$ units when supplier 1 is OFF and supplier 2 just starts his ON period.)

With this definition of a cycle, we would need to compute the new expected cycle length and cycle cost. Unfortunately, in this case we cannot extend the analysis presented in Section 1.1 in a straightforward manner using the definition of the new cycle. For example, the expression for cycle length $T_{10}$ is not analogous to the expression for the cycle length $T_{11}$ in (4). In other words,

$$
T_{10} \neq \begin{cases}q_{10}, & \text { if } \zeta\left(q_{10}\right)=10 \\ & \quad\left[\text { with probability } P_{10,10}\left(q_{10}\right)\right], \\ q_{10}+T_{l y}, & \text { if } \zeta\left(q_{10}\right) \neq 10 \\ & \quad\left[\text { with probability } P_{10, l}\left(q_{10}\right)\right] .\end{cases}
$$

This follows because the event $\zeta\left(q_{10}\right)=10$ does not necessarily start a new cycle, since the process may have already spent some time in state 10 by the time inventory drops to $r$ after $q_{10}$ time units. Hence, a proper definition of the expected cycle length (and cost) would require an analysis different from the one presented in Section 1.1.

There is, however, one special case where we can easily compare the $(q, r)$ and $\left(\mathbf{q}^{(3)}, r\right)$ policies: If we assume that $s_{1}=s_{2}=1$, i.e., the $\mathrm{ON}$ periods for both suppliers are distributed exponentially, then the cycle for the $\left(q^{(3)}, r\right)$ policy is defined similar to the cycle of the $(q, r)$ policy due to the memoryless property of the exponential distribution. Thus, both policies can be easily compared.

For this state-dependent $M[M] / M[M]$ model with $N=4$ states $\{11,10,01,00\}$, cycles would start whenever the inventory level increases to $q_{11}+r$ and both suppliers are available (i.e., the process is in state 11 ). We can then write:

$$
T_{11}= \begin{cases}q_{11}, & \text { if } \zeta\left(q_{11}\right)=11 \\ & {\left[\text { with probability } P_{11,11}\left(q_{11}\right)\right],} \\ q_{11}+T_{\imath \jmath}, & \text { if } \zeta\left(q_{11}\right) \neq 11 \\ & {\left[\text { with probability } P_{11, l]}\left(q_{11}\right)\right] .}\end{cases}
$$

so that $\bar{T}_{11}=q_{11}+\sum_{l \jmath \neq 11} \bar{T}_{\imath \jmath} P_{11, u l}\left(q_{11}\right)$ from (5). For $i j \neq$ 00 , we obtain:

$$
T_{l \jmath}=\left\{\begin{array}{lc}
q_{l \jmath}, & \text { if } \zeta\left(q_{l \jmath}\right)=11 \\
& {\left[\text { with probability } P_{l \jmath, 11}\left(q_{\imath \jmath}\right)\right],} \\
q_{l \jmath}+T_{k m}, & \text { if } \zeta\left(q_{\imath \jmath}\right)=k m \neq 11 \\
& {\left[\text { with probability } P_{l \jmath, k m}\left(q_{\imath \jmath}\right)\right],}
\end{array}\right.
$$

and for $i j=00$, the same expression in (7) applies. The expected cycle length $\bar{T}_{11}$ is now a function of three variables $\mathbf{q}^{(3)}$, and it can be computed in a manner similar to (3) after suitably redefining the $\mathbf{t}$ vector.

The expected cycle cost $\bar{C}_{11}$ is a function of four variables $\left(q^{(3)}, r\right)$, and it, too, can be computed using the procedure explained in Theorem 2. We note, however, that the cost of ordering and holding inventory in an interval that starts with $q_{i j}+r$ units and ends with $r$ units is $c\left(q_{i j}, r\right)=K+h q_{i j}^{2} / 2+h q_{i j} r, i j=11,10,01$. Thus, after suitably redefining the $c$ vector and solving for $\bar{C}_{11}$, the average cost function becomes:

$\mathscr{C}^{(3)}\left(\mathbf{q}^{(3)}, r\right)=\frac{\bar{C}_{11}\left(\mathbf{q}^{(3)}, r\right)}{\bar{T}_{11}\left(\mathbf{q}^{(3)}\right)}$,

which can be minimized with respect to the four decision variables $\left(\mathbf{q}^{(3)}, r\right)$.

We now discuss an example and compare the $(q, r)$ and $\left(\mathbf{q}^{(3)}, r\right)$ policies for the state-dependent $M[M] / M[M]$ model.

Example 4. We use the same cost parameter values as in Example 2; i.e., $K=\$ 200 /$ order, $h=\$ 100 /$ unit/time, and $b=\$ 500 /$ unit. Supply availability parameters are chosen as $\lambda_{1}=3 / 10, \lambda_{2}=1 / 4, \mu_{1}=1$, and $\mu_{2}=3 / 4$, so that the expected ON/OFF durations are $E\left(X_{1}\right)=10 / 3, E\left(X_{2}\right)=4$, $E\left(Y_{1}\right)=1$, and $E\left(Y_{2}\right) 4 / 3$. We solved the same set of eight problems discussed in Example 2. The results (to two significant digits) are presented in Table III, where the last column 12 provides a comparison of the objective function values of both policies. In this example we see that the suboptimal $(q, r)$ policy that we have chosen in this paper performs well compared to the better policy $\left(\mathbf{q}^{(3)}, r\right)$. Although the optimal values of $(q, r)$ and $\left(\mathbf{q}^{(3)}, r\right)$ are not necessarily equal, the optimal $\mathscr{C}^{*}$ and $\mathscr{b}^{(3) *}$ are so close that the deterioration in the objective function resulting from the use of the suboptimal policy becomes negligibly small, as shown in column 12 of Table III. This result can be attributed to the flatness of the objective functions around their optimal values. Thus, even if the suboptimal $(q, r)$ policy is implemented, the minimum value of the 
Table III

Comparison of the $(q, r)$ and $\left(\mathbf{q}^{(3)}, r\right)$ Policies for Varying $K, h$, and $b$

\begin{tabular}{cccccccccccc}
\hline$K$ & $h$ & $b$ & $q^{*}$ & $r^{*}$ & $\mathscr{C}^{*}$ & $q_{11}^{*}$ & $q_{10}^{*}$ & $q_{01}^{*}$ & $r^{*}$ & $\mathscr{C}^{(3) *}$ & \multicolumn{2}{c}{$\left[\mathscr{C}^{*} / \mathscr{C}^{(3) *}-1\right] \times$} \\
$(1)$ & $(2)$ & $(3)$ & $(4)$ & $(5)$ & $(6)$ & $(7)$ & $(8)$ & $(9)$ & $(10)$ & $(11)$ & $(12)$ \\
\hline 200 & 100 & 500 & 2.04 & 0.00 & 204.66 & 2.01 & 2.07 & 2.06 & 0.00 & 204.65 & $0.004 \%$ \\
& & 1,000 & 2.11 & 0.00 & 212.24 & 2.06 & 2.18 & 2.14 & 0.00 & 212.18 & $0.028 \%$ \\
& 300 & 500 & 1.16 & 0.00 & 350.14 & 1.15 & 1.17 & 1.16 & 0.00 & 350.13 & $0.003 \%$ \\
400 & & 1,000 & 1.19 & 0.00 & 362.14 & 1.16 & 1.23 & 1.21 & 0.00 & 362.03 & $0.038 \%$ \\
& 100 & 500 & 2.85 & 0.00 & 285.31 & 2.84 & 2.86 & 2.85 & 0.00 & 285.30 & $0.003 \%$ \\
& & 1,000 & 2.90 & 0.00 & 290.91 & 2.88 & 2.94 & 2.91 & 0.00 & 290.90 & $0.003 \%$ \\
& 300 & 500 & 1.63 & 0.00 & 490.09 & 1.63 & 1.63 & 1.63 & 0.00 & 490.08 & $0.002 \%$ \\
& 1,000 & 1.66 & $\mathbf{0 . 0 0}$ & 499.38 & 1.64 & 1.68 & 1.67 & 0.00 & 499.32 & $0.012 \%$ \\
\hline
\end{tabular}

Note: Some $r^{*}$ values are positive but $<0.001$.

resulting objective is not likely to be too far from that of the better policy.

In the second half of this example, we present the solution of 16 additional problems where we vary the supply uncertainty parameters $\lambda_{1}, \mu_{1}, \lambda_{2}$, and $\mu_{2}$ that cover a wide range, while keeping the monetary parameters $K, h$, and $b$ at their base levels of 200,100 , and 500 , respectively. The results are presented in Table IV, where we see again that the suboptimal policy that we have chosen performs well compared to the better policy $\left(\mathbf{q}^{(3)}, r\right)$.

As indicated at the beginning of this section, modeling the $\left(\mathbf{q}^{(3)}, r\right)$ policy for the nonexponential, i.e., Erlangian, case would require a redefinition of the regenerative cycle and reconstruction of the model from scratch. However, when this model is built, the expected cycle time and cycle cost expressions should be computable in a manner similar to the expressions (3) and (9), respectively, with the $\mathbf{Q}$ matrix and $\mathbf{t}$ and $\mathbf{c}$ vectors having different forms. Thus, the average cost objective function would still have a structure that is similar to the original model. Additionally, since the objective function appears to be flat around the optimal solution for these type of order quantity/reorder point problems, it is likely that the suboptimal policy $(q, r)$ will still perform well compared to the better policy $\left(\mathbf{q}^{(3)}\right.$, $r$ ) for the Erlangian case.

Ideally, one would need to use the machinery of dynamic programming to prove the optimality of a particular policy used in any inventory model. (For example, see Bartmann and Beckmann 1992.) Although the $\left(\mathbf{q}^{(3)}, r\right)$ policy dominates the $\left(\mathbf{q}^{(2)}, r\right)$ and $(q, r)$ policies, dynamic programming may reveal that there may exist yet another policy that is superior to $\left(\mathbf{q}^{(3)}, r\right)$. Identification of the optimal policy for the two-supplier environment discussed in this paper appears to be an open problem that should be studied in the future.

\section{SUMMARY AND EXTENSIONS}

This paper has analyzed an inventory problem where two suppliers in a market may sometimes be unavailable due to strikes, machine breakdowns, political upheavals, etc. We modeled the durations of availability of the suppliers using Erlangian random variables. The periods of unavailability were modeled using general random variables. The "method of stages" was used to transform the non-Markovian

Table IV

Comparison of the $(q, r)$ and $\left(\mathbf{q}^{(3)}, r\right)$ Policies for Varying $\lambda_{1}, \mu_{1}, \lambda_{2}$ and $\mu_{2}$

\begin{tabular}{|c|c|c|c|c|c|c|c|c|c|c|c|c|}
\hline $\begin{array}{l}\lambda_{1} \\
(1) \\
\end{array}$ & $\begin{array}{l}\mu_{1} \\
(2)\end{array}$ & $\begin{array}{l}\lambda_{2} \\
\text { (3) }\end{array}$ & $\begin{array}{l}\mu_{2} \\
\text { (4) }\end{array}$ & $\begin{array}{l}q^{*} \\
(5)\end{array}$ & $\begin{array}{l}r^{*} \\
(6)\end{array}$ & $\begin{array}{l}6^{*} \\
(7)\end{array}$ & $\begin{array}{l}q_{11}^{*} \\
(8)\end{array}$ & $\begin{array}{l}q_{10}^{*} \\
(9)\end{array}$ & $\begin{array}{l}q_{01}^{*} \\
(10)\end{array}$ & $\begin{array}{c}r^{*} \\
(11)\end{array}$ & $\begin{array}{l}\mathscr{C}^{(3) *} \\
(12) \\
\end{array}$ & $\begin{array}{c}\left(\mathscr{C}^{*} / \mathscr{C}^{(3) *}-1\right) \times 100 \\
(13)\end{array}$ \\
\hline \multirow[t]{4}{*}{0.1} & 0.2 & 0.05 & 0.1 & 2.07 & 0.00 & 224.78 & 2.00 & 2.20 & 2.09 & 0.00 & 224.64 & $0.062 \%$ \\
\hline & & & 5.0 & 2.00 & 0.00 & 200.09 & 2.00 & 2.00 & 2.00 & 0.00 & 200.09 & $0.000 \%$ \\
\hline & & 10 & 0.1 & 2.32 & 0.00 & 268.48 & 2.21 & 2.20 & 2.33 & 0.00 & 266.87 & $0.603 \%$ \\
\hline & & & 5.0 & 2.05 & 0.00 & 206.00 & 2.01 & 2.01 & 2.14 & 0.00 & 205.92 & $0.038 \%$ \\
\hline \multirow[t]{4}{*}{0.1} & 20 & 0.05 & 0.1 & 2.00 & 0.00 & 200.01 & 1.99 & 2.00 & 2.00 & 0.00 & 200.01 & $0.000 \%$ \\
\hline & & & 5.0 & 2.00 & 0.00 & 200.00 & 1.99 & 1.99 & 2.00 & 0.00 & 200.00 & $0.000 \%$ \\
\hline & & 10 & 0.1 & 2.00 & 0.00 & 200.03 & 1.99 & 1.99 & 1.99 & 0.00 & 200.03 & $0.000 \%$ \\
\hline & & & 5.0 & 2.00 & 0.00 & 200.01 & 2.00 & 2.00 & 1.97 & 0.00 & 200.01 & $0.000 \%$ \\
\hline \multirow[t]{4}{*}{10} & 0.2 & 0.05 & 0.1 & 2.31 & 0.00 & 264.22 & 2.06 & 3.43 & 2.08 & 0.00 & 260.44 & $1.451 \%$ \\
\hline & & & 5.0 & 2.00 & 0.00 & 200.27 & 1.99 & 1.98 & 2.00 & 0.00 & 200.26 & $0.005 \%$ \\
\hline & & 10 & 0.1 & 2.10 & 1.38 & 348.70 & 2.06 & 2.09 & 2.09 & 1.38 & 348.69 & $0.028 \%$ \\
\hline & & & 5.0 & 2.06 & 0.08 & 215.16 & 2.06 & 2.06 & 2.06 & 0.08 & 215.16 & $0.000 \%$ \\
\hline \multirow[t]{4}{*}{10} & 20 & 0.05 & 0.1 & 2.00 & 0.00 & 200.82 & 1.99 & 2.02 & 2.00 & 0.00 & 200.81 & $0.005 \%$ \\
\hline & & & 5.0 & 2.00 & 0.00 & 200.01 & 2.00 & 1.99 & 1.99 & 0.00 & 200.01 & $0.000 \%$ \\
\hline & & 10 & 0.1 & 2.02 & 0.00 & 202.42 & 2.03 & 2.02 & 2.02 & 0.00 & 202.42 & $0.000 \%$ \\
\hline & & & 5.0 & 2.01 & 0.00 & 201.32 & 2.01 & 2.01 & 2.01 & 0.00 & 201.32 & $0.000 \%$ \\
\hline
\end{tabular}

Note: Some $r^{*}$ values are positive but $<0.001$. 
stochastic process of suppliers' availability into a Markovian one. Renewal reward theorem was employed to construct the objective function of the long-run average cost per time. We discussed a special case where the problem was solved numerically. A second example was discussed for the case of asymptotic order quantity $q$. In this case, the constant limiting probabilities of the supplier availability stochastic process could be used to simplify the computation of the expected cost function. Finally, an alternative state-dependent inventory policy was introduced and compared to the $(q, r)$ policy used in the paper. It was found in several examples that although the alternative policy would dominate the $(q, r)$ policy, deterioration in the objective function value was negligibly small when the latter policy was employed.

We have used the Erlang distribution to represent the durations of the ON periods for both suppliers assuming that the coefficient of variation (COV) of these random variables does not exceed one. In practice, this would not create a serious difficulty, since for most problems the standard deviation of the periods of availability is likely to be smaller than their mean, i.e., COV $\leqslant 1$. However, it would be interesting to investigate the problem where $\mathrm{ON}$ periods have a general distribution without any restrictions on the magnitude of the COV. It may be possible to use the "supplementary variable", technique due to Cox (1955) to transform the non-Markovian process into a Markovian one. This transformation would require the use of an infinite number of state variables.

\section{ACKNOWLEDGMENT}

Research conducted while the second author was on sabbatical leave at the Department of Industrial Engineering, Bilkent University, Turkey, during Fall 1992. Financial support for the second author from the Natural Sciences and Engineering Research Council of Canada (A5872) is gratefully acknowledged. The authors would also like to acknowledge the associate editor/referee comments which have helped to improve the paper. Final thanks are due to Levent Kandiller and Nureddin Kurkavak for useful discussions on the practical implications of supply uncertainties.

\section{REFERENCES}

Bartmann, D. AND M. J. Beckmann. 1992. Inventory Control. Springer-Verlag, Berlin.

Bellman, R., R. E. Kalaba AND J. A. Lockett. 1966. Numerical Inversion of the Laplace Transform: Applications to Biology, Economics, Engineering and Physics. American Elsevier, New York.
CHAO, H. P. 1987. Inventory Policy in the Presence of Market Disruptions. Opns. Res. 35, 274-281.

Chao, H. P., S. W. Chapel, C. E. Clark, P. A. Morris, M. J. SANDling AND R. C. GRIMEs. 1989. EPRI Reduces Fuel Inventory Costs in the Electric Utility Industry. Interfaces 19, $48-67$.

Cox, D. R. 1955. The Analysis of Non-Markovian Stochastic Processes by the Inclusion of Supplementary Variables. Proc. Cambridge Philosophical Soc. 51, 33-41.

GerchaK, Y. And M. Parlar. 1990. Yield Randomness, Cost Tradeoffs and Diversification in the EOQ Model. Naval Res. Logist. 37, 341-354.

GotTFried, B. S. AND J. Weisman. 1973. Introduction to Optimization Theory. Prentice-Hall, Englewood Cliffs, New Jersey.

GuPTA, D. 1996. The $(Q, r)$ Inventory System with an Unreliable Supplier. INFOR, 34, 59-76.

Hadley, G. AND T. M. Whitin. 1963. Analysis of Inventory Systems. Prentice-Hall, Englewood Cliffs, N.J.

JERRI, A. J. 1985. Introduction to Integral Equations with Applications. Marcel Dekker, New York.

KLEINROCK, L. 1975. Queueing Systems, Volume 1: Theory. John Wiley, New York.

Meyer, R. R., M. H. Rothkopf and S. F. SMrth. 1979. Reliability and Inventory in a Production-Storage System. Mgmt. Sci. 25, 799-807.

Monzadeh, K. and P. Aggarwal. 1992. Analysis of a Production/Inventory System Subject to Random Disruptions. Working Paper, School of Business, University of Washington.

Nahmias, S. 1993. Production and Operations Analysis. Second Edition, Irwin, Homewood, Illinois.

PARLAR, M. 1997. Continuous-Review Inventory Problem with Random Supply Interruptions. Euro. Inl. Oper. Res. 99, 366-385.

Parlar, M. AND D. Berkin. 1991. Future Supply Uncertainty in EOQ Models. Naval Res. Logist. 38, 107-121.

Parlar, M. AND D. PERry. 1996. Inventory Models of Future Supply Uncertainty with Single and Multiple Suppliers. Naval Res. Logist. 43, 191-210.

Press, W. H., B. P. Flannery, S. A. Teukolsky and W. T. VetTERling. 1989. Numerical Recipes: The Art of Scientific Computing. Cambridge University Press, Cambridge.

Ross, S. 1983. Stochastic Processes. John Wiley, New York.

SIlver, E. A. 1981. Operations Research in Inventory Management: A Review and Critique. Opns. Res. 29, 628-645.

Silver, E. A. ANd R. Peterson. 1985. Decision Systems for Inventory Management and Production Planning. Second Edition, John Wiley \& Sons, New York.

Van LaArhoven, P. J. M. And E. H. L. Aarts. 1987. Simulated Annealing: Theory and Applications. D. Reidel, Dordrecht. 
Copyright 1997, by INFORMS, all rights reserved. Copyright of Operations Research is the property of INFORMS: Institute for Operations Research and its content may not be copied or emailed to multiple sites or posted to a listserv without the copyright holder's express written permission. However, users may print, download, or email articles for individual use. 